\title{
Spectral properties of the post-merger gravitational-wave signal from binary neutron stars
}

\author{
Kentaro Takami, ${ }^{1}$ Luciano Rezzolla,${ }^{1}$ and Luca Baiotti ${ }^{2}$ \\ ${ }^{1}$ Institut für Theoretische Physik, Max-von-Laue-Straße 1, 60438 Frankfurt, Germany \\ ${ }^{2}$ Graduate School of Science, Osaka University, 560-0043 Toyonaka, Japan
}

\begin{abstract}
Extending previous work by a number of authors, we have recently presented a new approach in which the detection of gravitational waves from merging neutron star binaries can be used to determine the equation of state of matter at nuclear density and hence the structure of neutron stars. In particular, after performing a large number of numerical-relativity simulations of binaries with nuclear equations of state, we have found that the post-merger emission is characterized by two distinct and robust spectral features. While the high-frequency peak was already shown to be associated with the oscillations of the hypermassive neutron star produced by the merger and to depend on the equation of state, we have highlighted that the low-frequency peak is related to the merger process and to the total compactness of the stars in the binary. This relation is essentially universal and provides a powerful tool to set tight constraints on the equation of state. We here provide additional information on the extensive analysis performed, illustrating the methods used, the tests considered, as well as the robustness of the results. We also discuss additional relations that can be deduced when exploring the data and how these correlate with various properties of the binary. Finally, we present a simple mechanical toy model that explains the main spectral features of the post-merger signal and can even reproduce analytically the complex waveforms emitted right after the merger.
\end{abstract}

PACS numbers: 04.25.D-, 04.25.dk, 04.30.Db, 26.60.Kp

\section{INTRODUCTION}

As a series of advanced detectors such as LIGO [1], Virgo [2], and KAGRA [3] is going to become operational in the next five years, we are likely to witness soon the first direct detection of gravitational waves (GWs). The sources that are likely to be detected first are obviously the strongest ones and, in particular, the inspiral and post-merger of neutron-star binaries or neutron-star-black-hole binaries, and binary black holes. Although an uncertainty as large as 3 orders of magnitude accompanies the estimates coming from populationsynthesis models, it is widely assumed that binary neutron star (BNS) mergers will represent the most common source, with an expected detection rate of $\sim 40 \mathrm{yr}^{-1}$ [4].

In addition to being excellent sources of GWs, the merger of a BNS system represents also the most attractive scenario to explain the phenomenology associated with short gamma-ray bursts (SGRBs). While suggested already in the 1980s [5, 6], the recent progress of numerical simulations [7-11] and the wealth of additional astronomical simulations (see [12] for a recent review) has put this scenario on firm ground. Over the years, these simulations have shown that the merger of BNSs inevitably leads to the formation of a massive metastable object, which can either collapse promptly or survive up to a fraction of a second emitting large amounts of gravitational radiation. If the NSs comprising the binary have large magnetic fields and extended magnetospheres the inspiral can be accompanied by a precursor electromagnetic signal [13], while the merger can lead to instabilities [14, 15] and to the formation of magnetically confined jet structures once a torus is formed around the black hole (BH) $[16,17]$. The coincident detection of an electromagnetic and GW emission would represent the most convincing evidence of the link between SGRBs and BNSs.

Any GW signal from a binary including a neutron star will contain important signatures of the equation of state (EOS) of matter at nuclear densities. A first signature is represented by the tidal corrections to the orbital phase; these are reasonably well understood analytically [18-20] and can be tracked accurately with advanced high-order numerical codes [21, 22]. A second signature is instead related to the post-merger phase, where the object formed by the merger [most likely a hypermassive neutron star (HMNS)] can emit GWs in a narrow frequency range before collapsing to a $\mathrm{BH}[8,23,24]$.

The first detailed evidence that it is possible to extract information about the EOS of nuclear matter by carefully investigating the spectral properties of the post-merger signal was provided by Bauswein and Janka [25, 26]. After performing a large number of simulations using a smoothed particle hydrodynamics code solving the Einstein field equations assuming conformal flatness and employing a GW backreaction scheme within a post-Newtonian approximation, they pointed out the presence of a peak at high-frequency in the spectrum (dubbed $f_{\text {peak }}$ ) and showed it correlated with the properties of the EOS, e.g., with the radius of the maximum-mass nonrotating configuration (see also Ref. [27] for a subsequent generalrelativistic study). The correlation found was rather tight, but restricted to binaries having all the same total mass of $2.7 M_{\odot}$. It was later recognized that $f_{\text {peak }}$ corresponds to a fundamental fluid mode with $m=2$ of the HMNS [28] and that the information from this frequency could also be used to set constraints on the maximum mass of the system and hence on the EOS [29].

Following this bulk of work, we have recently presented in Ref. [30] a new approach in which the detection of GWs from merging BNSs can be used to determine the EOS of matter at nuclear density and hence the structure of neutron stars. More specifically, making use of a large number of accurate numerical-relativity simulations of binaries with nuclear EOSs and different masses, we have shown that the postmerger emission is characterized by two distinct and robust spectral features. The high-frequency of these two peaks is the 
same already discussed in Refs. [25, 26], but we have also revealed that the correlations with the stellar properties depend on the total mass of the system and hence on the EOS. On the other hand, we have also shown that the low-frequency peak is related to the merger process and related to the total compactness of the stars in the binary. This relation is essentially universal and provides a powerful tool to set tight constraints on the EOS.

The purpose of this paper is to provide additional information on the extensive analysis performed and presented in [30], illustrating the methods used, the tests considered, as well as the robustness of the results. Besides describing in greater detail the spectral properties discussed above, we also discuss additional correlations that either have been already discussed in the literature, e.g., in Refs. [31, 32], or that are presented here for the first time. An important aspect of this paper is also that of presenting a simple mechanical toy model that can explain rather intuitively the main spectral features of the postmerger signal and shed light on the physical interpretation of the origin of the various peaks. Despite its crudeness, the toy model can even reproduce the complex waveforms emitted right after the merger, hence possibly opening the way to an analytical modeling of a part of the signal that has been so far considered impossible to model but with numerical-relativity calculations. The waveforms are freely available for download and further analysis [33].

The paper is organized as follows. Section II is dedicated to the numerical setup used to carry out the simulations and discusses our mathematical formulation, the structure and extent of the numerical domain, the EOSs used, and the set of BNSs considered. Section III deals instead with the analysis of the GW signal, concentrating in particular on the approach followed to compute in an automated way the spectral properties of the post-merger signal. The validation of the numerical results is instead discussed in Sec. IV, where we consider how the grid resolution or the thermal contribution to the EOS influence the results. Section V is the scientific core of the paper, and after a global overview of the results (Sec. V A), we present a simple explanation for the origin of the $f_{1}$ and $f_{3}$ frequency peaks (Sec. V B), as well as a number of correlations that the relevant frequencies in the system, i.e., $f_{\max }$ (the frequency at amplitude maximum) $f_{1}, f_{2}$, and $f_{3}$, have with the stellar properties (Sec. V C-V F). We also discuss in Sec. V G how to exploit the knowledge of the spectral properties to constrain the EOS of nuclear matter, either in idealized conditions or in more realistic ones (Sec. V H). While the conclusions are reserved for Sec. VI, we dedicate Appendix A to illustrate the details of the toy model.

We use a spacelike signature $(-,+,+,+)$ and a system of units in which $c=G=M_{\odot}=1$ unless stated differently.

\section{NUMERICAL SETUP}

Much of the numerical setup used in these simulations has been presented in greater detail in other papers [8, 34, 35], and for compactness we will review here only the basic aspects, referring the interested reader to the papers above for additional information. All of our simulations have been performed in full general relativity using a fourth-order finitedifferencing code McLachlan [36, 37], which solves a conformal traceless formulation of the Einstein equations [3840 ], with a " $1+\log$ " slicing condition and a "Gamma-driver" shift condition [41, 42]. At the same time, the generalrelativistic hydrodynamics equations are solved using the finite-volume code Whisky [43], which has been extensively tested in simulations involving the inspiral and merger of BNSs [8, 19, 34, 44].

We note that differently from previous works of ours, e.g., [31], but also from those of other authors, e.g., [45], we have not employed as approximate Riemann solver the Marquina flux formula [46], which has been revealed to be problematic when used in conjunction with nuclear-physics EOSs approximated as piecewise polytropes (see discussion in Sec. II B). Instead, we have employed the Harten-Lax-van Leer-Einfeldt (HLLE) [47] approximate Riemann solver [47], which is less accurate but more robust, in conjunction with a piecewise parabolic method (PPM) for the reconstruction of the evolved variables [48]. We also note that although we have recently developed a new code, i.e., WhiskyTHC, able to reach high-order convergence [21, 22], we have not employed it here mostly because our focus is on the post-merger dynamics, where the large scale shocks reduce the accuracy of both codes to first order, and for which Whi sky appears to be slightly more robust.

For the time integration of the coupled set of the hydrodynamic and Einstein equations we have used the method of lines (MOL) in conjunction with an explicit fourth-order Runge-Kutta method [49]. In all our simulations we prescribe a Courant-Friedrichs-Lewy (CFL) factor of 0.35 to compute the size of the time step.

\section{A. Grid structure and extent}

To place the outer boundary at sufficiently large distances and guarantee at the same high spatial resolution near the neutron stars, we employ an adaptive-mesh refinement (AMR) approach which follows closely the one adopted in [8, 50]. In practice, the grid hierarchy is handled by the Carpet meshrefinement driver [51]. It implements vertex-centered mesh refinement, also known as the box-in-box method, and allows for regridding during the calculation as well as multiple grid centers. As discussed in Ref. [51], within this approach a number of grids with different resolutions, called refinement levels, overlay each other, and are nested in such a way that the coarsest grid has the largest extent and the finest grid the smallest extent. The time step on each grid is set by the Courant condition and so by the spatial grid resolution for that level. Boundary data for finer grids are calculated with spatial prolongation operators employing fifth-order polynomials and with prolongation in time employing second-order polynomials.

During the inspiral phase, the positions of the centers of the two highest-resolution grids are determined by the location of the maximum of the rest-mass density. One grid is placed at 
the grid point correspondent to $\rho_{\max }$ and the other at the $\pi$ symmetric point, namely the point obtained by a rotation of $180 \mathrm{deg}$ around the $z$ axis for equal-mass binaries. The grid hierarchy is composed of six refinement levels and a $2: 1$ refinement factor for successive levels. The grid resolution varies from $\Delta h_{5}=0.15 M_{\odot}$ (i.e., $\simeq 221 \mathrm{~m}$ ) for the finest level to $\Delta h_{0}=4.8 M_{\odot}$ (i.e., $\simeq 7.1 \mathrm{~km}$ ) for the coarsest level, whose outer boundary is at $514 M_{\odot}$ (i.e., $\simeq 759 \mathrm{~km}$ ). Initially, the number of grid points across the linear dimension of a star is of the order of 100, while these become roughly twice when the merger has taken place and a HMNS has been formed.

The whole grid is set up to be symmetric with respect to the $(x, y)$ plane both for equal- and unequal-mass binaries, with a reflection symmetry across the $z=0$ plane to reduce computational costs. Additionally, a $\pi$-symmetry condition across the $x=0$ plane is adopted in the case of equal-mass binaries to further reduce the cost. The boundary conditions are chosen to be radiative for the metric to prevent gravitational waves from scattering back into the grid, and static for the hydrodynamical variables. No matter reaches the outer boundary over the time scale of the simulations.

\section{B. Equations of state}

We model the stars with nuclear-physics EOSs by considering five different EOS models: i.e., APR4 [52], ALF2 [53], SLy [54], H4 [55], and GNH3 [56]. We note that all of these EOSs satisfy the current observational constraint on the observed maximum mass in neutron stars, i.e., $2.01 \pm 0.04 M_{\odot}$ obtained for the pulsar PSR J0348+0432 [57]. In order to cover a range in stellar compactness that is larger than the one spanned by the above five nuclear EOSs, we have also considered an additional EOS given by a single polytrope, with pressure expressed as $p_{\mathrm{c}}=K \rho^{\Gamma}$, where $\rho, K$, and $\Gamma$ are the rest-mass density, the polytropic constant, and the polytropic exponent, respectively (see, e.g., Ref. [49] for details).

Instead of using tables for the various EOSs, we have found it simpler and more convenient to reproduce each EOS in terms of a number $N$ of piecewise polytropes [58]. More specifically, the "cold" and nuclear-physics contribution to each EOS is obtained after expressing the pressure and specific internal energy $\epsilon_{\mathrm{c}}$ in the rest-mass density range $\rho_{i-1} \leq$ $\rho<\rho_{i}$ as

$$
\begin{aligned}
& p_{\mathrm{c}}=K_{i} \rho^{\Gamma_{i}}, \\
& \epsilon_{\mathrm{c}}=\epsilon_{i}+K_{i} \frac{\rho^{\Gamma_{i}-1}}{\Gamma_{i}-1},
\end{aligned}
$$

In addition, we impose the continuity of pressure and specific internal energy through the following conditions

$$
\begin{aligned}
K_{i+1} & =K_{i} \rho_{i}^{\Gamma_{i}-\Gamma_{i+1}} \\
\epsilon_{i+1} & =\epsilon_{i}+K_{i} \frac{\rho_{i}^{\Gamma_{i}-1}}{\Gamma_{i}-1}-K_{i+1} \frac{\rho_{i}^{\Gamma_{i+1}-1}}{\Gamma_{i+1}-1},
\end{aligned}
$$

for $i=1, \ldots, N-1$.

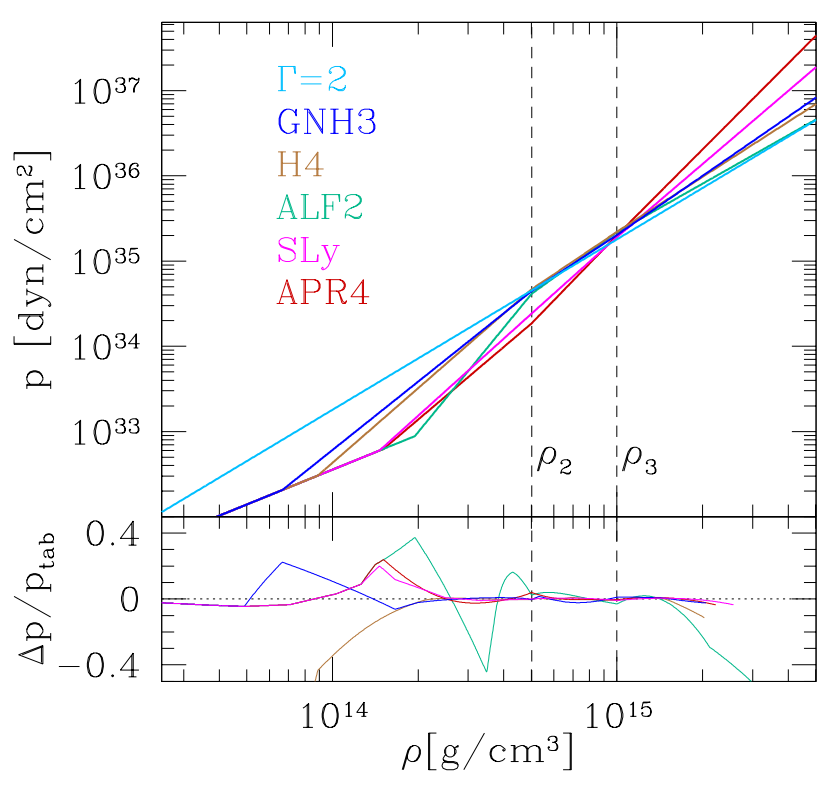

FIG. 1. Top panels: pressure as a function of the rest-mass density for the EOSs in Table I when represented in terms of piecewise polytropes. Bottom panel: the corresponding relative error $\Delta p / p_{\text {tab }}$, where $\Delta p \equiv p_{\text {tab }}-p$ and $p_{\text {tab }}$ is the pressure in tabulated form. The tabulated EOSs for H4 and ALF2 are provided by [58], while the others are from the LORENE library [59].

As shown in Ref. [58], $N=4$ pieces, three of which describe the high-density core and one the crustal region, are sufficient to reproduce to good precision most of the EOSs. In the top panel of Fig. 1 we show the behavior of the various EOSs used as well as when represented as piecewise polytropes, while the bottom panel measures the relative error. Also reported in Table I are the various parameters used for the piecewise polytropes.

The cold nuclear-physics EOSs are adequate to represent the state of the neutron-star matter prior to the merger. After contact, however, large shocks will develop, considerably increasing the internal energy. To account for this additional heating we could use hot nuclear-physics EOSs, which provide information about the dependence of the pressure and density also as a function of temperature. In practice, however, the number of such EOSs is still pretty limited, thus preventing us from doing a systematic investigation. As a result, we follow [25, 26] and account for thermal effects by using a so-called "hybrid EOS" [49], that is, by adding to the cold part an ideal-fluid component that accounts for the shock heating [60]. In practice the total pressure and specific internal energy are expressed as

$$
\begin{aligned}
& p=p_{\mathrm{c}}+p_{\mathrm{th}}, \\
& \epsilon=\epsilon_{\mathrm{c}}+\epsilon_{\mathrm{th}},
\end{aligned}
$$

where $p_{\mathrm{c}}, \epsilon_{\mathrm{c}}$ are given by Eqs. (1) and (2), while the "thermal" 


\begin{tabular}{c|c|ccc|c|cc}
\hline EOS & $N$ & $\Gamma_{2}$ & $\Gamma_{3}$ & $\Gamma_{4}$ & $\begin{array}{c}\rho_{1} \\
{\left[\mathrm{~g} / \mathrm{cm}^{3}\right]}\end{array}$ & $\begin{array}{c}M_{\max } \\
{\left[M_{\odot}\right]}\end{array}$ & $\begin{array}{c}R_{\max } \\
{[\mathrm{km}]}\end{array}$ \\
\hline$\Gamma=2$ & 1 & $\ldots$ & $\ldots$ & $\cdots$ & $\ldots$ & 1.8206 & 12.536 \\
GNH3 & 4 & 2.664 & 2.194 & 2.304 & $6.66038 \times 10^{13}$ & 1.9768 & 11.266 \\
H4 & 4 & 2.909 & 2.246 & 2.144 & $8.87824 \times 10^{13}$ & 2.0282 & 11.603 \\
ALF2 & 4 & 4.070 & 2.411 & 1.890 & $1.94771 \times 10^{14}$ & 1.9911 & 11.308 \\
SLy & 4 & 3.005 & 2.988 & 2.851 & $1.46231 \times 10^{14}$ & 2.0606 & 9.9349 \\
APR4 & 4 & 2.830 & 3.445 & 3.348 & $1.51201 \times 10^{14}$ & 2.2000 & 9.8733 \\
\hline
\end{tabular}

TABLE I. Parameters used to represent the nuclear-physics EOSs. In addition to the values reported in the table, we use the following values for the polytropic constants and indices: $K=123.647$, $K_{1} / c^{2}=3.99873692 \times 10^{-8}\left[\left(\mathrm{~g} / \mathrm{cm}^{3}\right)^{1-\Gamma_{1}}\right], \Gamma_{1}=1.35692395$. Similarly, different polytropic pieces are joined at the rest-mass densities $\rho_{2}=5.01187234 \times 10^{14}\left[\mathrm{~g} / \mathrm{cm}^{3}\right]$, and $\rho_{3}=10^{15}\left[\mathrm{~g} / \mathrm{cm}^{3}\right]$. The last two columns in the table report the gravitational mass and the radius of the maximum-mass model in the corresponding TolmanOppenheimerVolkoff (TOV) sequence.

part is given by

$$
\begin{aligned}
p_{\mathrm{th}} & =\rho \epsilon_{\mathrm{th}}\left(\Gamma_{\mathrm{th}}-1\right), \\
\epsilon_{\mathrm{th}} & =\epsilon-\epsilon_{\mathrm{c}},
\end{aligned}
$$

where $\Gamma_{\text {th }}$ is arbitrary but constrained mathematically to be $1 \leq \Gamma_{\text {th }} \leq 2$. Note that this hybrid-EOS approach is possible because the total specific internal energy $\epsilon$ is one of the evolved variables; hence its value is computed independently at each time level.

Before concluding, an important remark should be made about the use of piecewise polytropes in relativistic hydrodynamic simulations, especially when adopting higher-order reconstruction methods or sophisticated approximate Riemann solvers [49]. The prescription (1) for each piece of the polytrope, together with the junction conditions (3) across different pieces, guarantee that the pressure is everywhere a continuous function of the rest-mass density. Of course these conditions cannot guarantee that the derivatives of the pressure are themselves continuous. The accepted wisdom is that these discontinuities are harmless as they involve only a very limited number of cells during the evolution. However, whether this is actually true in practice depends crucially on the accuracy of the reconstruction algorithm and on the robustness of the Riemann solver employed. More importantly, the negative impact that these discontinuities may have on the evolution is often difficult to diagnose and hence avoid.

Figure 2 offers a very clear example of the risks that can accompany the use of a piecewise polytropic EOS by showing in the top panels two snapshots of the rest-mass density on the $(x, y)$ plane at a representative time of the evolution $(t \sim-12.50 \mathrm{~ms})$ of the binary H4-q10-M1350 with $\Gamma_{\text {th }}=1.36$, computed using either the Marquina approximate Riemann solver [46] (top left panel) or the HLLE Riemann solver [47] (top right panel). In both cases the reconstruction is made using the PPM prescription.

A quick comparison between the two distributions of the rest-mass density does not reveal any problematic behavior and indeed the solutions seem very much comparable, with

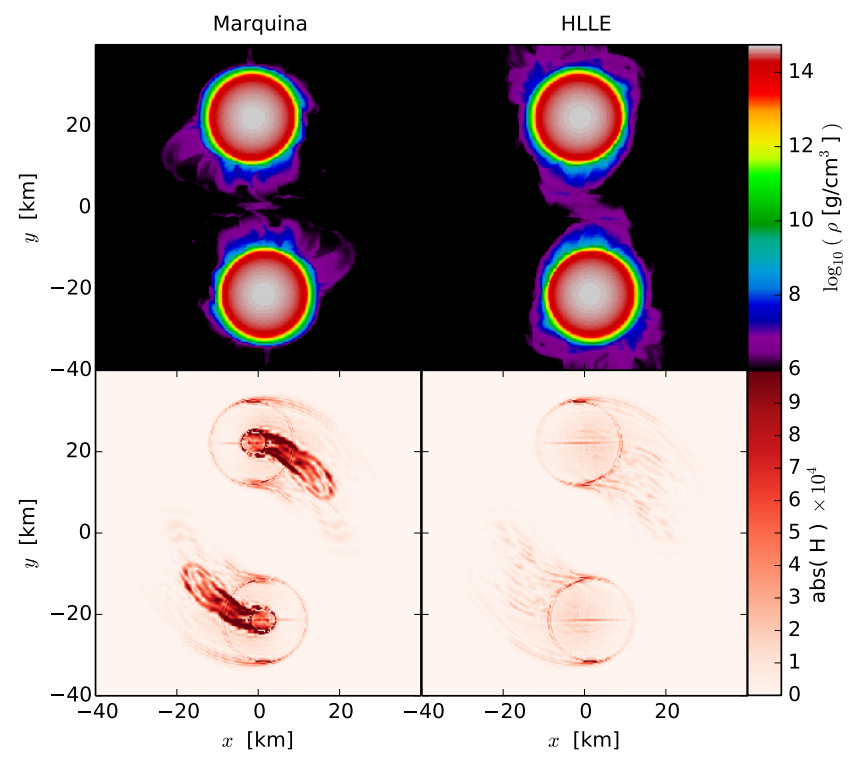

FIG. 2. Top panels: Snapshots of the rest-mass density on the $(x, y)$ plane at $t \simeq-12.50 \mathrm{~ms}$ in the evolution of the binary H4-q10-M1350 with $\Gamma_{\text {th }}=1.36$, computed using either the Marquina approximate Riemann solver (top left panel) or the HLLE Riemann solver (top right panel). Note that the two rest-mass distributions are rather similar. Bottom panels: Snapshots of the corresponding violations of the Hamiltonian constraints on the $(x, y)$ plane. Note that the Marquina solver leads to violations that are 1 or more orders of magnitude larger.

differences appearing mostly in the outer layers of the stars, close to the atmosphere and hence of little relevance in terms of the dynamics of the binary. This comparison is, however, rather deceptive and the conclusion is actually incorrect. The evolution with the Marquina solver, in fact, suffers from a much larger violation of the Hamiltonian constraint, with norm-2 values that can be 1 order of magnitude larger. This is illustrated in the bottom panels, which show the distributions of the corresponding violations of the Hamiltonian constraints on the $(x, y)$ plane. While both evolutions show sources of violations near the stellar surface (cf., outer rings in both solvers), the solution with the Marquina formula shows much larger violations in the central regions of the stars and, in particular, around the rest-mass density $\rho_{2}$, where the two most-central pieces of the EOS are joined.

A one-dimensional inspection of the rest-mass density would indeed reveal that the stars present rather macroscopic discontinuities around $\rho_{2}$ in the case of the solution with the Marquina solver, which are instead absent with the less accurate but more robust HLLE solver. As expected, the discontinuities appearing with the Marquina solver in the case of piecewise EOSs, disappear when a single polytropic EOS is used, with violations in the Hamiltonian constraint that are comparable to those of the HLLE solver.

To the best of our knowledge this is the first time that such a comparison is made and that these problems have been discussed in the context of BNS evolutions. It is possible that 


\begin{tabular}{|c|c|c|c|c|c|c|c|c|c|c|c|c|c|}
\hline Model & EOS & $q$ & $\begin{array}{c}\bar{M} \\
{\left[M_{\odot}\right]}\end{array}$ & $\begin{array}{c}\bar{R} \\
{[\mathrm{~km}]}\end{array}$ & $\begin{array}{c}M_{\mathrm{ADM}} \\
{\left[M_{\odot}\right]}\end{array}$ & $\begin{array}{c}\bar{M}_{\mathrm{b}} \\
{\left[M_{\odot}\right]}\end{array}$ & $\bar{M} / \bar{R}$ & $\begin{array}{l}f_{\text {orb }} \\
{[\mathrm{Hz}]}\end{array}$ & $\begin{array}{c}J \\
{\left[M_{\odot}^{2}\right]}\end{array}$ & $\bar{I} / \bar{M}^{3}$ & $\overline{k_{2}}$ & $\lambda / \bar{M}^{5}$ & $\begin{array}{l}f_{\text {cont }} \\
{[\mathrm{Hz}]}\end{array}$ \\
\hline GAM2-q10-M1350 & & 1 & 1.350 & 6.774 & 2.6748 & 1.4336 & & 282.47 & 7.3039 & & & 755.3 & 980.58 \\
\hline GAM2-q10-M1375 & $=2$ & 1 & & & 2.7239 & 1.4623 & 12183 & 284.37 & 7.5274 & 20.203 & & 2361.6 & I. \\
\hline & -2 & 1 & & & & & & 286.25 & & & & & \\
\hline & $=2$ & 1 & 425 & & 8221 & 1.5200 & & 288.10 & 7.9838 & & & & 1038.9 \\
\hline & $=2$ & 1 & 450 & .313 & 2.8712 & 1.5491 & 3125 & 289.93 & 8.2167 & 17.612 & & 0.3 & 059.7 \\
\hline & NH3 & 1 & .250 & 7 & 4780 & 1.3464 & 58 & 273.29 & 6.4067 & 18.890 & & & 262 \\
\hline & H3 & & 275 & 0 & 5271 & 1.3756 & & 275.38 & 6.61 & 18.237 & & & 127 \\
\hline & & 1 & & & 2.5763 & 1.4050 & & 277.44 & & & & & \\
\hline & & 1 & & & & & & & & & & & \\
\hline & NH3 & 1 & 350 & 7 & 2.6746 & 1.4641 & 468 & 281.58 & 7.2766 & 16.450 & & & 317.3 \\
\hline & NH3 & 0.92593 & 1.300 & 3.797 & 2.5763 & 1.4052 & 0.13912 & 277.51 & 6.8255 & 17.607 & & 1445.2 & 1289.9 \\
\hline & & & 250 & & 4780 & 1.3506 & & 273.25 & 6.4058 & & & & 302.1 \\
\hline & & 1 & 275 & & 2.5271 & 1.3799 & & 275.40 & & & & & \\
\hline & & & & & & & & & & & & & \\
\hline & & & 325 & & & 1.4390 & & 279.60 & & & & & \\
\hline & & & 350 & 0 & 2.6746 & 1.4687 & 0.14711 & 281.61 & 7.2770 & 16.344 & & & 1350.6 \\
\hline M1225 & & & 225 & 2.252 & 4288 & 1.3373 & & 271.00 & 6.1923 & & & & \\
\hline & & 1 & & 6 & & 3672 & & & & & & & \\
\hline & & & & & & & & & & & & & \\
\hline & LF2 & & 1.300 & 12.319 & 2.5763 & 1.4272 & & 277.26 & & & & & 1529.0 \\
\hline & LF2 & & 1.325 & 2.337 & 2.6254 & 1.4574 & & 279.36 & 7.0475 & & & & \\
\hline & & & 250 & & 2.4779 & 1.3720 & & & & & & & \\
\hline & & 1 & & & & & & & & & & & \\
\hline & & 1 & & & & & & & & & & & \\
\hline & & & & & & & & & & & & & \\
\hline 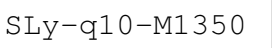 & & 1 & 1.350 & 1.465 & 2.6745 & 1.4946 & & & & & & & \\
\hline & & & & & & & & & & & & & \\
\hline & & 1 & & & & & & & & & & & \\
\hline & & 1 & 0 & 11.067 & & 1.4399 & & 277.24 & & 12.436 & & .19 & 1795.5 \\
\hline & & 1 & 1.325 & 11.07 & 2.6254 & 1.4709 & & 279.31 & & 12.070 & & & \\
\hline & PR4 & 1 & 1.350 & 11.079 & 2.6746 & 1.5020 & 0.17992 & 281.37 & 7.2665 & 11.720 & & 321.78 & 1826.7 \\
\hline APR4-q10-M1375 & APR4 & 1 & 1.375 & 11.084 & 2.7237 & 1.5334 & 0.18317 & 283.39 & 7.4924 & 11.387 & 0.088786 & 287.10 & 1842.3 \\
\hline
\end{tabular}

TABLE II. All models and their properties. The various columns denote the gravitational mass ratio $q \equiv M_{1} / M_{2}$ at infinite separation, the average gravitational mass $\bar{M}$ at infinite separation, the average radius $\bar{R}$ at infinite separation, the Arnowitt-Deser-Misner (ADM) mass $M_{\mathrm{ADM}}$ of the system at initial separation, the baryon mass $\bar{M}_{\mathrm{b}}$, the compactness $\mathcal{C} \equiv \bar{M} / \bar{R}$, the orbital frequency $f_{\text {orb }}$ at the initial separation, the total angular momentum $J$ at the initial separation, the dimensionless moment of inertia $\bar{I} / \bar{M}^{3}$ at infinite separation, the $\ell=2$ dimensionless tidal Love number $\bar{k}_{2}$ at infinite separation, the dimensionless tidal deformability $\lambda / \bar{M}^{5}$ defined by $\lambda \equiv 2 \bar{k}_{2} \bar{R}^{5} / 3$, and the contact frequency $f_{\text {cont }}=\mathcal{C}^{3 / 2} /(2 \pi \bar{M})[61]$. The quantities with a bar are defined as averages, i.e., $\bar{A} \equiv\left(A_{1}+A_{2}\right) / 2$.

the violations of the constraints are made more severe by the fact that the violations have zero propagation velocities and that once formed they cannot leave the computational domain. In this case, the use of alternative formulations of the equations, such as the recently proposed conformal and covariant Z4 formulation (CCZ4) [62], which has been shown to lead to reduced violations of the constraints [63], are likely to yield improved evolutions in the case of higher-order methods or Riemann solvers. A more systematic investigation is needed to address this point.

\section{Initial data}

In order to generate the initial data for quasiequilibrium irrotational BNSs with these EOSs, we use the multidomain spectral-method code LORENE [64] under the assumption of a conformally flat spacetime metric. We consider a rather small initial coordinate separation of the stellar centers of $45 \mathrm{~km}$ for all models, since we here focus mostly on the GW emission from the merger and post-merger stages.

Clearly, if the HMNS is short-lived, it is hard to obtain reliable statistical properties of the GWs from the HMNS, 
and thus construct empirical correlations. As a result, we have selected initial-data models with masses such that the HMNS has a lifetime $t_{\mathrm{HMNS}} \geq 5000 M_{\odot} \approx 24.63 \mathrm{~ms}$. For each EOS, we have considered five equal-mass binaries with average (gravitational) mass at infinite separation in the range $\bar{M} \equiv\left(M_{1}+M_{2}\right) / 2=(1.275-1.375) M_{\odot}$ for the APR4 EOS, $(1.225-1.325) M_{\odot}$ for the ALF2 EOS, $(1.250-1.350) M_{\odot}$ for the GNH3, H4, and SLy EOSs, and $(1.350-1.450) M_{\odot}$ for the $\Gamma=2$ polytrope. Higher masses would lead to HMNSs that collapse to a BH-torus system well before a time $5000 M_{\odot} \approx 25 \mathrm{~ms}$. In addition, we have also considered two unequal-mass binaries with $\bar{M}=1.300 M_{\odot}$ and mass ratio $q \simeq 0.93$ for the GNH3 and SLy EOSs. Detailed information on all the models and the properties used in our evolutions is collected in Table II, but it is worth remarking that all in all we have considered 32 different binaries with different EOSs and mass ratios. Together with the work of Ref. [27], this represents the largest sample of simulations of BNSs with nuclear-physics EOSs.

\section{ANALYSIS OF THE GRAVITATIONAL-WAVE SIGNAL}

\section{A. Definitions and conventions}

We extract the GW signal at different surfaces of constant coordinate radius using the Newman-Penrose formalism, so that the GW polarization amplitudes $h_{+}$and $h_{\times}$are related to the Weyl curvature scalar $\psi_{4}$ by (see Sec. IV of Ref. [8] for details)

$$
\ddot{h}_{+}-\mathrm{i} \ddot{h}_{\times}=\psi_{4}=\sum_{\ell=2}^{\infty} \sum_{m=-\ell}^{\ell} \psi_{4}^{\ell m}{ }_{-2} Y_{\ell m}(\theta, \varphi),
$$

where the overdot indicates a time derivative and we have introduced the (multipolar) expansion of $\psi_{4}$ in spin-weighted spherical harmonics [65] of spin weight $s=-2$. In our analysis we consider only the $\ell=m=2$ mode, which is the dominant one both in the inspiral and after the merger; i.e., we assume $^{1}$

$$
h_{+, \times}=\sum_{\ell=2}^{\infty} \sum_{m=-\ell}^{\ell} h_{+, \times-2}^{\ell m} Y_{\ell m}(\theta, \varphi) \approx h_{+, \times-2}^{22} Y_{22}(\theta, \varphi),
$$

where ${ }_{s} Y_{\ell m}(\theta, \varphi)$ are the spin-weighted spherical harmonics. Unless explicitly stated, we consider the optimally oriented case, for which ${ }_{-2} Y_{22}(\theta=0, \varphi=0) \approx 0.63$. The GWs are sampled in time at a rate of $\Delta t=1.68 M_{\odot} \simeq 8.27 \times 10^{-3} \mathrm{~ms}$, equivalent to a sampling rate of $\simeq 121 \mathrm{kHz}$.

Following previous work [31], and in order to evaluate the GWs in the same phase for all models, we align the waveforms at the time of merger, which we set to be $t=0$ and define to be correspondent to the time when the GW amplitude

$$
|h| \equiv\left(h_{+}^{2}+h_{\times}^{2}\right)^{1 / 2}
$$

\footnotetext{
${ }^{1}$ Note that for simplicity in Ref. [66] we have used $h_{+, \times}=h_{+, \times}^{22}$.
}

reaches its first maximum. As a result, for all binaries we consider $\mathrm{GW}$ signals in the time interval $t \in$ $[-1500,5000] M_{\odot} \approx[-7.39,24.63] \mathrm{ms}$, which includes GWs from approximately $3-4$ orbits before merger.

The instantaneous frequency of the GW is then computed as [31]

$$
f_{\mathrm{GW}} \equiv \frac{1}{2 \pi} \frac{d \phi}{d t}
$$

where $\phi=\arctan \left(h_{\times} / h_{+}\right)$is the phase of the complex gravitational waveform. As a consequence, the GW frequency at the time of merger, or equivalently the frequency at maximum amplitude $^{2}$, is given by

$$
f_{\text {max }} \equiv f_{\mathrm{GW}}(t=0) .
$$

We next define the power spectral density (PSD) of the effective amplitude as

$$
\tilde{h}(f) \equiv \sqrt{\frac{\left|\tilde{h}_{+}(f)\right|^{2}+\left|\tilde{h}_{\times}(f)\right|^{2}}{2}},
$$

with

$$
\tilde{h}_{+, \times}(f) \equiv\left\{\begin{array}{ll}
\int h_{+, \times}(t) e^{-i 2 \pi f t} d t & (f \geq 0) \\
0 & (f<0)
\end{array},\right.
$$

and where the + and $\times$ indices refer to the two polarization modes. Finally, the signal-to-noise ratio (SNR) is computed as

$$
\mathrm{SNR} \equiv\left[\int_{0}^{\infty} \frac{\left|2 \tilde{h}(f) f^{1 / 2}\right|^{2}}{S_{h}(f)} \frac{d f}{f}\right]^{1 / 2}
$$

where $S_{h}(f)$ is the noise PSD of the GW detector under consideration (i.e., Advanced LIGO [67], or the Einstein Telescope (ET) [68, 69]).

\section{B. Spectral-properties detection}

An important aspect of our analysis consists in extracting from the PSD of the post-merger signal those spectral features that can be correlated with the physical properties of the binary system. Such an extraction could be done by a simple visual inspection of the spectra as the peaks are generally quite evident. Yet, doing so for computing the exact position of the various frequencies would introduce an inevitable bias that would be difficult to reproduce systematically. As a result, following the spirit of Ref. [70], we adopt a more algorithmic approach in which the PSDs are first fitted using a prescribed functional and then estimated from the fit. The identification of the peak obtained in this way is a robust measure in the sense that it matches very well the one that would

\footnotetext{
${ }^{2}$ In Ref. [31] this frequency was denoted as $f_{\text {peak }}$.
} 


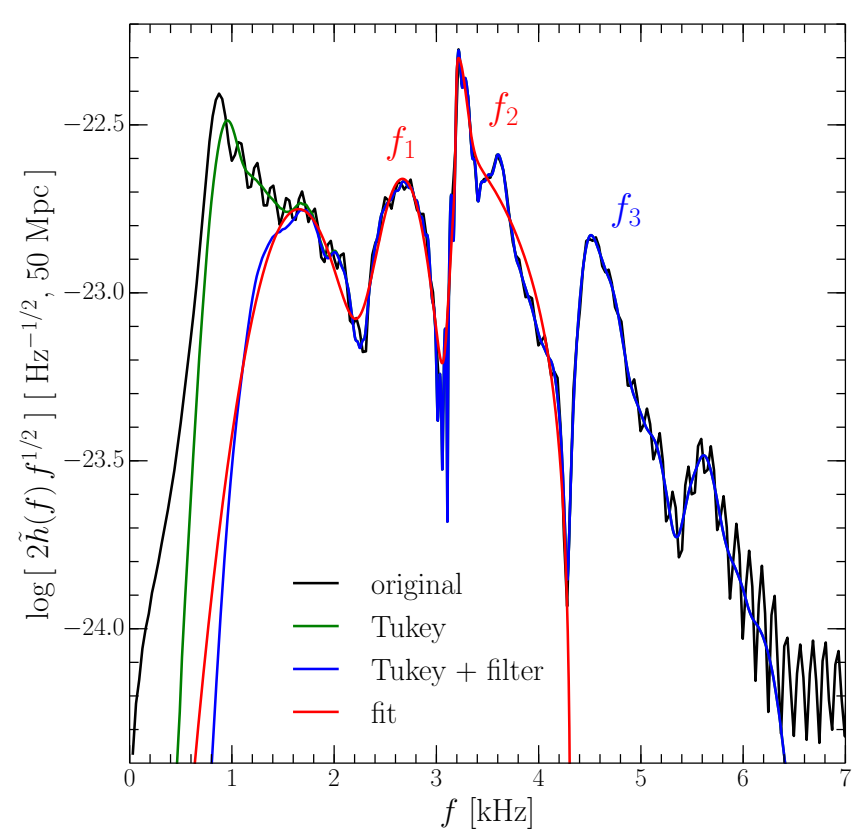

FIG. 3. PSD $2 \tilde{h}(f) f^{1 / 2}$ relative to the binary APR $4-q 10-M 1375$. Indicated with a black line is the full PSD, while the green line shows the PSD after the application of a Tukey window, and the blue line the PSD filtered with a high-pass Butterworth filter. Finally, shown in red is the fit made to capture the peak frequencies $f_{1}$ and $f_{2}$.

be done "by eye" and guarantees that other authors can reproduce it straightforwardly. More specifically, our detection of the spectral properties can be summarized with the following steps:

(1) We first apply to the GW time series a symmetric timedomain Tukey window function with alpha parameter 0.25 [71, 72]. This window affects the initial and final $\sim 4 \mathrm{~ms}$ of the waveform and is applied to compute a PSD without the artificial noise due to the cutting off of the waveform. A representative example of the application of this window is shown in Fig. 3, which reports the PSD of the binary APR4-q10-M1375. Shown with a black line is the PSD relative to the full waveform, while the PSD computed after the Tukey window is shown with a green line. Clearly, no differences are visible for $f \gtrsim 1.5 \mathrm{kHz}$, and a cutoff to the power is introduced for $f \gtrsim 6.5 \mathrm{kHz}$.

(2) We apply a fifth-order high-pass Butterworth filter [73] with a cutoff frequency at $f_{\text {cut }} \sim f_{\mathrm{GW}}(t=$ $\left.-1500 M_{\odot}\right)+0.5 \mathrm{kHz} \sim[(0.65-0.75)+0.5] \mathrm{kHz}$. This is done to remove the contribution coming from the inspiral and thus provide a PSD which is easier to model analytically. The resulting filtered PSD is shown in Fig. 3 with a blue line.

(3) We fit the filtered PSD $\tilde{h}(f)$ obtained after step (2) with the analytic function

$$
\begin{aligned}
\mathcal{S}(f)= & A_{1} e^{-\left(f-F_{1}\right)^{2} / W_{1}^{2}}+\mathcal{S}_{2}(f) \\
& +A_{0} e^{-\left(f-F_{0}\right)^{2} / W_{0}^{2}}
\end{aligned}
$$

where the first term captures the low-frequency peak at the merger, the second term captures the high-frequency peak at HMNS, while the last term is used to model the very-low frequency peak corresponding to the inspiral. Although Ref. [70] used a trapezoid function to approximate the high-frequency peak in an ideal-fluid EOS, we use here a combination of a trapezoid and a Gaussian function as it produces a better match to the functional form of the PSD in the case of nuclear-physics EOSs. The explicit form of this part is given by

$$
\mathcal{S}_{2}(f)=A_{2 G} e^{-\left(f-F_{2 G}\right)^{2} / W_{2 G}^{2}}+A(f) \gamma(f),
$$

where

$$
\begin{aligned}
A(f) \equiv & \frac{1}{2 W_{2}}\left[\left(A_{2 b}-A_{2 a}\right)\left(f-F_{2}\right)\right. \\
& \left.+W_{2}\left(A_{2 b}+A_{2 a}\right)\right], \\
\gamma(f) \equiv & \left(1+e^{-\left(f-F_{2}+W_{2}\right) / s}\right)^{-1} \\
& \times\left(1+e^{\left(f-F_{2}-W_{2}\right) / s}\right)^{-1} .
\end{aligned}
$$

The 14 parameters, $A_{1}, F_{1}, W_{1}, A_{2 a}, A_{2 b}, F_{2}, W_{2}, s$, $A_{2 G}, F_{2 G}, W_{2 G}, A_{0}, F_{0}, W_{0}$, are computed via a nonlinear least-squares fitting. An example of the resulting fit is shown also in Fig. 3 with a red line. Clearly, the fitting procedure has provided a very good representation of the low- and high-frequency peaks. Similarly good results are obtained also with other EOSs.

(4) Finally, we associate the two spectral features of the PSD which are our interest here, i.e., $f_{1}$ and $f_{2}$, with the values of the coefficient $F_{1}$ and with the frequency average of the function $\mathcal{S}_{2}{ }^{3}$

$$
f_{1} \equiv F_{1}, \quad f_{2} \equiv \frac{\int \mathcal{S}_{2}(f) f d f}{\int \mathcal{S}_{2}(f) d f} .
$$

\section{VALIDATION OF THE NUMERICAL RESULTS}

As mentioned above, the purpose of this work is mainly to highlight the relation between the spectral properties of the post-merger signal and the physical properties of the merging binary. In this sense, here we are not particularly interested to make sure that this relation is quantitatively accurate; far

\footnotetext{
${ }^{3}$ Because the notation in the literature has been evolving and a bit confusing, we remark that what we indicate as $f_{2}$ was marked as $f_{\text {peak }}$ in Ref. [25] and as $f_{2}$ in Ref. [28]. This latter notation is becoming the standard one.
} 

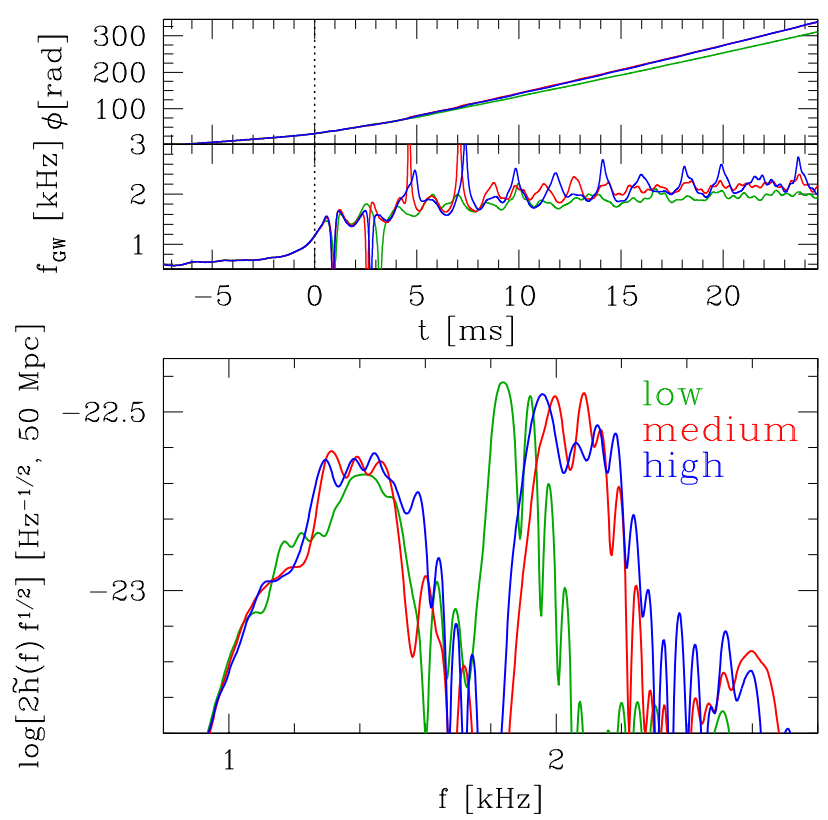

FIG. 4. Top panel: evolution of the instantaneous GW phase $\phi$ for the binary GAM2-q10-M1400 when computed at low (green), medium (red), and high (blue) resolution, respectively. Middle panel: instantaneous frequency $f_{\mathrm{GW}}$ of the GW signal for the same binary and at the same resolutions. Bottom panel: corresponding PSDs $2 \tilde{h}(f) f^{1 / 2}$ for optimally oriented sources at a distance of $50 \mathrm{Mpc}$.

more important to us is that our analysis captures the correct qualitative behavior. Nevertheless, it is important to assess how the results obtained also depend on choices that are under our control, such as the grid resolution and the choice of the polytropic index $\Gamma_{\text {th }}$ for the thermal part of the EOS. While a discussion of these aspects is rarely done in the literature, in what follows we discuss both of these aspects in detail.

\section{A. Dependence on grid resolution}

Performing accurate measurements of the convergence order as the ones presented in [22] or in [11] is clearly not possible when considering the very large number of binaries and EOSs considered here. Hence, rather than determining the convergence order, we have here explored whether our results are in a regime of consistency, i.e., the truncation error decreases with increasing resolution [49]. While a much weaker requirement than convergence, an overall consistency guarantees that the numerical errors are bound and decreasing. We have therefore considered as a representative example the evolution of the single-polytropic BNS GAM2-q10-M1 400 when performed at three different spatial resolutions of $\left(\Delta h_{5}\right)_{\text {low }}=0.175 M_{\odot} \simeq 258 \mathrm{~m}$ (low resolution), $\left(\Delta h_{5}\right)_{\text {med }}=0.150 M_{\odot} \simeq 221 \mathrm{~m}$ (medium resolution), and $\left(\Delta h_{5}\right)_{\text {high }}=0.125 M_{\odot} \simeq 185 \mathrm{~m}$ (high resolution).

The results of this comparison are shown in Fig. 4, where the top panel reports evolution of the GW phase, with the green, red, and blue lines referring to the low, medium, and high resolutions, respectively. Shown instead in the middle panel is the instantaneous frequency $f_{\mathrm{GW}}$ of the GW signal. It is apparent that the phase evolution is already well captured by the middle resolution, with differences with respect to the high resolution that are $\Delta \phi \simeq 0.9 \mathrm{rad}$ at the end of simulation, that is, with a fractional change $\Delta \phi / \phi \simeq 0.3 \%$. Similarly, the evolutions of the three instantaneous frequencies are essentially indistinguishable up to the merger (cf., dotted vertical line), so that the peak frequency $f_{\max }$ varies at most of $\simeq 12 \mathrm{~Hz}$ (i.e., $\simeq 1 \%$ ) when going from the low to the high resolution and with differences of $\lesssim 7 \mathrm{~Hz}$ (i.e., $\simeq 0.6 \%$ ) between the medium and high resolutions. On the other hand, the three instantaneous frequencies are clearly different after the merger. This behavior, which cannot be removed by a simple time shift, is a clear indication that the resolutions used are not sufficiently high to guarantee that the instantaneous frequency is a robust quantity after the merger.

The bottom panel of Fig. 4 shows a different type of information as it focuses on the time-integrated spectral density $2 \tilde{h}(f) f^{1 / 2}$ for the three different resolutions and is relative to a source at $50 \mathrm{Mpc}$. A rapid inspection of the figure is sufficient to conclude that while the low resolution may be sufficient to capture the behavior of the low-frequency $\left(f_{1}\right)$ peak around $1.4 \mathrm{kHz}$, it does not provide a consistent description of the high-frequency $\left(f_{2}\right)$ peak around $2.1 \mathrm{kHz}$. Fortunately, however, the medium resolution is sufficient to obtain a reliable measure of $f_{2}$; more precisely the differences in the estimates of the $f_{1}$ and $f_{2}$ frequencies between the medium and high resolution runs vary by $\lesssim 20 \mathrm{~Hz}$ and $\lesssim 35 \mathrm{~Hz}$, respectively. Because these uncertainties are below the ones at which our results are valid, i.e., $\sim 200 \mathrm{~Hz}$ (see discussion in Sec. VD), we conclude that the medium resolution of $\left(\Delta h_{5}\right)_{\text {med }}=0.150 M_{\odot}$ is sufficient for our scopes. As a side remark we note that this choice is also a forced one. The results presented here, in fact, have been collected over more than one year of calculations with an expenditure of $\sim 3 \times 10^{6}$ CPU hours. Computational costs larger than these are simply not available to us at the moment.

\section{B. Dependence on the thermal component}

As discussed in Sec. II B, because we are not using here hot nuclear-physics EOSs, the thermal effects are accounted for by hybrid EOS in which an ideal-fluid contribution is added to the total pressure. The choice of the corresponding adiabatic index $\Gamma_{\text {th }}$ is somewhat arbitrary, with only a mathematical constraint that it lays in the range $1 \leq \Gamma_{\text {th }} \leq 2$ (but see discussion in [49]), while finite-temperature EOSs such as the Shen EOS [74] suggest that values $\Gamma_{\text {th }} \sim 1.6-2.0$ are preferable [75]. Because $\Gamma_{\text {th }}$ regulates the amount of thermal pressure produced after the merger, and hence the survival time of the HMNS, it is natural to ask how the spectral properties of the post-merger signal depend on the value chosen for $\Gamma_{t h}$.

Figure 5 collects the results of this analysis and in particular it shows the PSDs relative to the binary H4-q10-M1300 


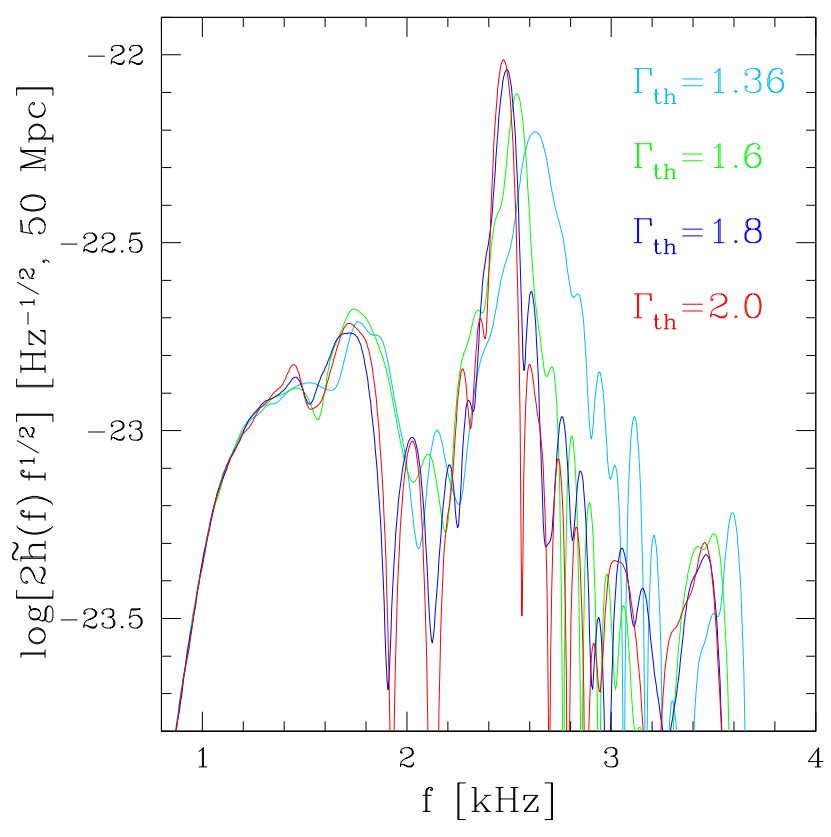

FIG. 5. Behavior of the PSDs $2 \tilde{h}(f) f^{1 / 2}$ when the adiabatic exponent of the thermal contribution to the EOS is set to be $\Gamma_{\text {th }}=$ $1.36,1.6,1.8$, and 2.0 for the binary $\mathrm{H} 4-\mathrm{q} 10-\mathrm{M} 1300$. The PSDs have been computed for waveforms over the time interval $t \in$ $[-1500,2800] M_{\odot} \approx[-7.39,13.79] \mathrm{ms}$.

when four different values of the thermal component, i.e., $\Gamma_{\text {th }}=1.36,1.6,1.8$, and 2.0 are used. Note that because smaller values of $\Gamma_{\text {th }}$ favor the collapse to a black hole, the PSDs are computed over the common interval in which a HMNS is present, i.e., $t \in[-1500,2800] M_{\odot} \approx$ $[-7.39,13.79] \mathrm{ms}$.

As one would expect given that the $f_{1}$ peak is built right after the merger, its value is not much affected by the choice for $\Gamma_{\mathrm{th}}$, with the differences that are $\lesssim 90 \mathrm{~Hz}$. The value of the $f_{2}$ peak, on the other hand, is somewhat more sensitive for small values of $\Gamma_{\text {th }}$, but is remarkably robust for $\Gamma_{\text {th }} \gtrsim 1.8$, that is, in the most realistic range. To be more quantitative, the frequency of the $f_{2}$ peak varies by $\lesssim 50 \mathrm{~Hz}$ when $1.6 \leq \Gamma_{\text {th }} \leq 2.0$, with a maximum variation of $\lesssim 130 \mathrm{~Hz}$ when the more extreme case of $\Gamma_{\mathrm{th}}=1.36$ is included. In the light of these results, but also to maintain a consistency with the results presented in Ref. [25] and with our singlepolytrope sequence GAM2-q10, we have assumed $\Gamma_{\text {th }}=2$ as the standard thermal contribution in all of our simulations.

\section{RESULTS}

\section{A. Global overview}

As discussed above and as listed in Table II, we have computed a total of 32 binaries having six different EOSs and five different masses. Before making a detailed discussion on the correlations between the spectral features, i.e., $f_{1}$ and $f_{2}$, and the physical properties of the merging stars, it is useful to have a global overview of the results so that it is easier to capture the general behaviors of the HMNS and the most obvious features of the gravitational-wave signal.

Figure 6 collects all of the waveforms for the equal-mass models with nuclear-physics EOSs as evolved at the reference medium resolution. The waveforms are organized in a tabular form in which each row refers to a given EOS, while each column concentrates on a given initial mass. The different EOSs are distinguished by different colors and we will adopt this color coding also for all the subsequent plots.

When scrolling through the different panels, some common features become quite evident. First, the pre-merger signal and the post-merger one are in frequency ranges that are considerably different. This is not surprising given the significant difference in compactness in the system before and after the merger, but points to the fact that the phenomenology of the signal will necessarily have to be split into two parts. Second, a transient period of 1-3 ms separates these two signals and represents the time needed by systems to readjust itself from the quasiperiodic inspiral over to the quasiperiodic rotation of the HMNS. As we will comment later on, despite being very short, this transient contains very important information and is where the power in the $f_{1}$ peak is built. Third, the transient is followed by a period of $\sim 10 \mathrm{~ms}$ during which oscillations at different frequencies are present and which anticipates the stage in which the HMNS has reached a "stationary" state and is rotating at a frequency that increases only slowly with time as a result of GW losses. Fourth, the amplitude of the signal in the post-merger phase increases with mass. This is rather easy to understand as larger masses will also lead to larger changes in the quadrupole moment; the only exception to this behavior is represented by the APR4 EOS, which is particularly soft at high densities and hence yields an HMNS which rapidly becomes almost axisymmetric. Finally, the amplitude in the post-merger decays and the rate at which this happens depends inversely on the mass and on the stiffness, so that soft EOSs show a large damping in amplitude (e.g., binary APR4-q10-M1325), while stiff ones show a much smaller damping (e.g., binary GNH3-q10-M1300). This too is easy to understand and reflects the fact that HMNSs with larger masses (and not ultrasoft) will tend to maintain the bar deformation for longer times.

Next, we can explore an equivalent global view of the GW signal, but when measured in the frequency domain. More specifically, we show in Fig. 7 the PSDs $2 \widetilde{h}(f) f^{1 / 2}$ for the same binaries considered in Fig. 6 and organized in the same manner when the binaries are at a distance of $50 \mathrm{Mpc}$. Note that the colored dashed lines refer to the full time series (i.e., including the inspiral), while the solid lines refer to the high-passed filtered data as discussed in Sec. III B. In each panel we indicate with a filled circle the "contact frequency" $f_{\text {cont }} \equiv \mathcal{C}^{3 / 2} /(2 \pi \bar{M})[61]$, where $\mathcal{C} \equiv \bar{M} / \bar{R}$ is the average compactness, $\bar{R} \equiv\left(R_{1}+R_{2}\right) / 2$, and $R_{1,2}$ are the radii of the nonrotating stars associated with each binary [30]. Finally, reported with solid lines are the sensitivity curves of Advanced LIGO and ET (green and light-blue lines, respectively). 


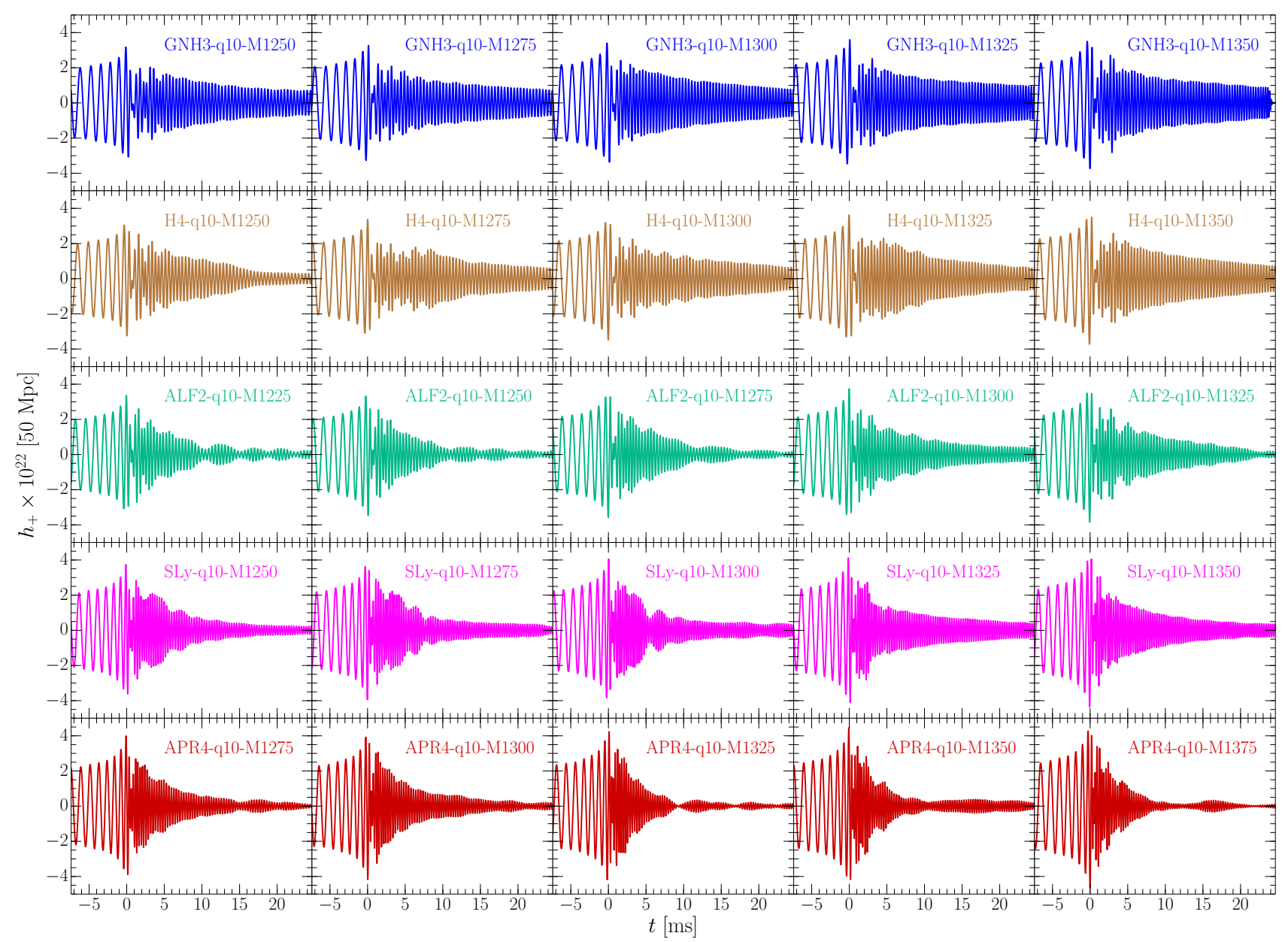

FIG. 6. Gravitational waveforms for all the binaries with equal masses and nuclear-physics EOSs as evolved at the reference medium resolution. Each row refers to a given EOS, while each column concentrates on a given initial mass. The different EOSs are distinguished by different colors, and we will adopt this color coding also for all the subsequent plots; more details on the various binaries are shown in Table II.

Also in the frequency domain, a rapid scan of the panels allows one to discern the most important features. First, and as discussed by several authors [8, 23-27, 66, 70], all PSDs show a clear and strong peak, i.e., the $f_{2}$ peak, which, at these distances, can be 1 order of magnitude or more above the sensitivity curve of the Advanced LIGO detectors. This peak is clearly related to the rotation of the bar-deformed HMNS and corresponds, in a corotating frame, to a (quadrupolar) $\ell=m=2$ mode moving at a positive pattern speed in the prograde direction $[28]^{4}$. As we will comment later in Sec. VE, this mode can be seen to correlate with a number of properties of the stars comprising the binary, although this dependence is different for different EOSs and is "universal" only at a fixed mass.

All of the panels also show the presence of a low-frequency peak, i.e., the $f_{1}$ peak, which has already been discussed in

\footnotetext{
${ }^{4}$ As customary, the prograde direction is the direction of rotation of the HMNS as seen in an inertial frame.
}

detail in Ref. [28], where it was indicated as $f_{-}$. This peak always has a power smaller than that of $f_{2}$ and it can happen that if the EOS is particularly soft (e.g., as for the binary APR4-q10-M1275) or if the mass is particularly small (e.g., as for the binary SLy-q10-M1250), it is hard to distinguish it from the background. However, because the peak is also sitting in a region where the sensitivity of detectors is higher, it will be detectable at these distances with a SNR smaller but comparable to that of the $f_{2}$ peak (cf., Table III). As remarked in [30], this peak is is produced by the nonlinear oscillations of the two stellar cores that collide and bounce repeatedly right after the merger. More important, as we will comment later in Sec. V D, this mode correlates tightly with the stellar compactness $\mathcal{C}$ in a way that is essentially universal, that is independent of the EOS.

In addition to the $f_{1}$ and $f_{2}$ peaks, the PSDs also show the presence of an additional peak at frequencies higher than $f_{2}$ (see top left panel of Fig. 7). We have dubbed this peak as $f_{3}$ (in Ref. [28] it was instead indicated as $f_{+}$) and its value is approximated as $f_{3} \sim 2 f_{2}-f_{1}$ with a precision of about 


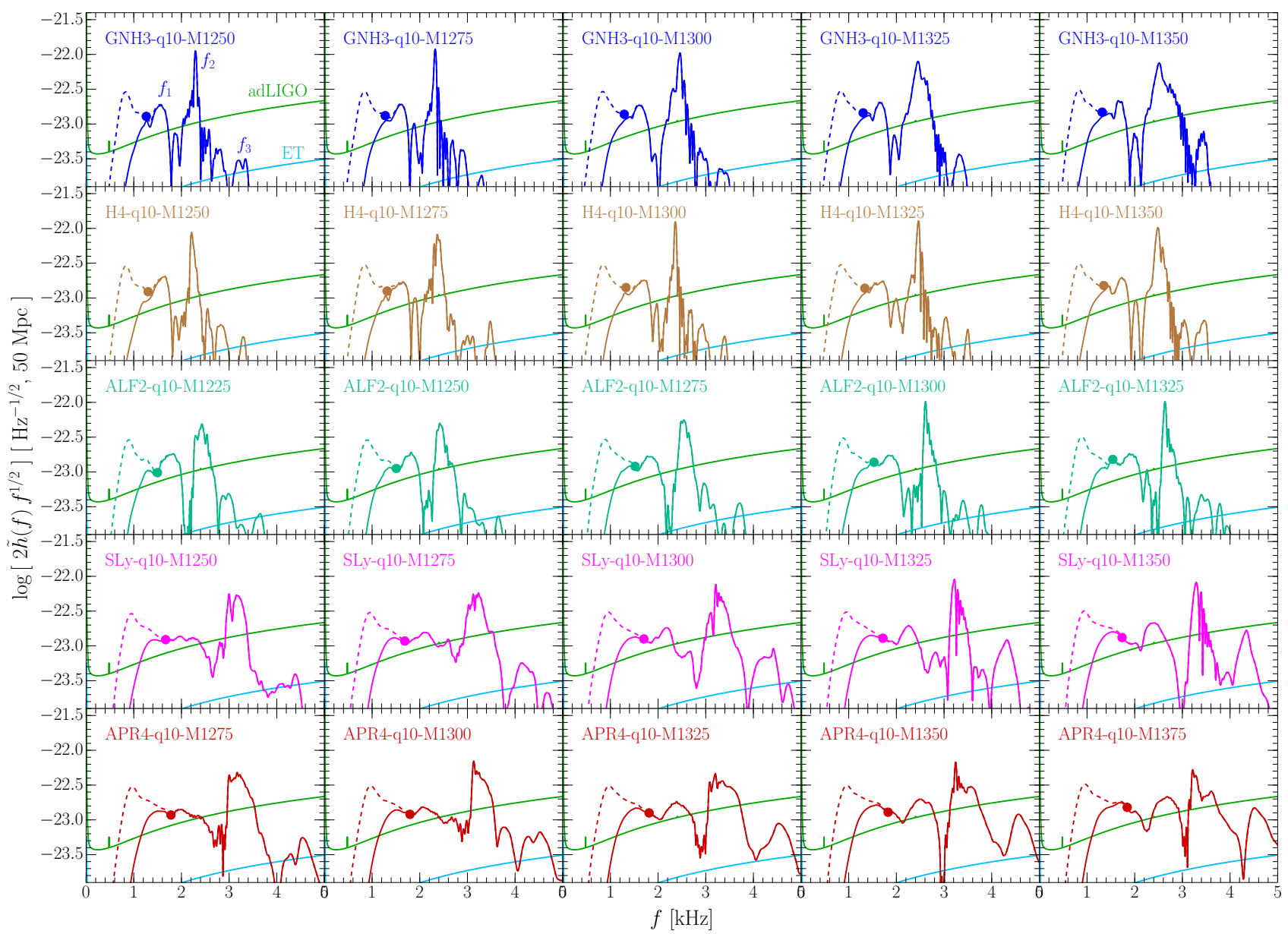

FIG. 7. PSDs $2 \tilde{h}(f) f^{1 / 2}$ for the equal-mass binaries with nuclear-physics EOS shown in Fig. 6 . Solid lines of different colors refer to the high-passed waveforms, while the dashed lines refer to the full waveforms. Indicated with colored circles are the various contact frequencies $f_{\text {cont }}$, while the curves of Advanced LIGO and ET are shown as green and light-blue lines, respectively.

$10 \%$. While equally interesting and potentially containing additional information on the merging system, this peak is the one with the least power of the three and is usually located at very high frequencies, always below the sensitivity curve of Advanced LIGO. Hence, more sensitive detectors, such as ET, will be needed to observe this spectral feature even at moderate distances.

\section{B. On the origin of the $f_{1}$ and $f_{3}$ peaks}

It has so far been unclear what is the actual physical origin of the two frequency peaks $f_{1}$ and $f_{3}$. It is possible to attribute $f_{1}$ to a nonlinear interaction between the quadrupole and quasiradial modes [28]; similarly, it is possible that $f_{3}$ is an overtone or the result of the nonlinear interaction of the $f_{2}$ mode with other nonquasiradial modes [28]. These perturbative suggestions are given substance by the fact that the $f_{2}$ peak is, to first approximation, the average of the $f_{1}$ and $f_{3}$ frequencies, and it is well known that if a perturbed system has eigenfrequencies $f_{i}$, the nonlinearity of the equa- tions will also produce modes at frequencies $f_{i} \pm f_{j}$ (see Sec. 28 of Ref. [76]). On the other hand, the amplitudes of these nonlinear couplings are usually found to be considerably smaller than the originating eigenfrequencies (see the discussion in [77]), and our PSDs show instead that the amplitudes in the $f_{1}-f_{3}$ peaks vary by a factor of few and not of orders of magnitude.

On the other hand, a different interpretation is possible on the origin of these modes. In this interpretation, which we suggest here, they are simply produced by the GW emission due to the nonlinear oscillations of the two stellar cores that collide and bounce repeatedly. Animations of the few milliseconds following the instant when the stars get in contact, in fact, show that the HMNS attains a quasistationary configuration with a marked bar-mode deformation only $\sim 5 \mathrm{~ms}$ after the merger. On the other hand, the object produced after the contact is far more irregular and the two stellar cores collide and bounce repeatedly as a result of the strong rotation and very high densities. This is shown in Fig. 8 for the representative binary $A L F 2-q 10-M 1325$. The figure contains four different panels reproducing the rest-mass density 


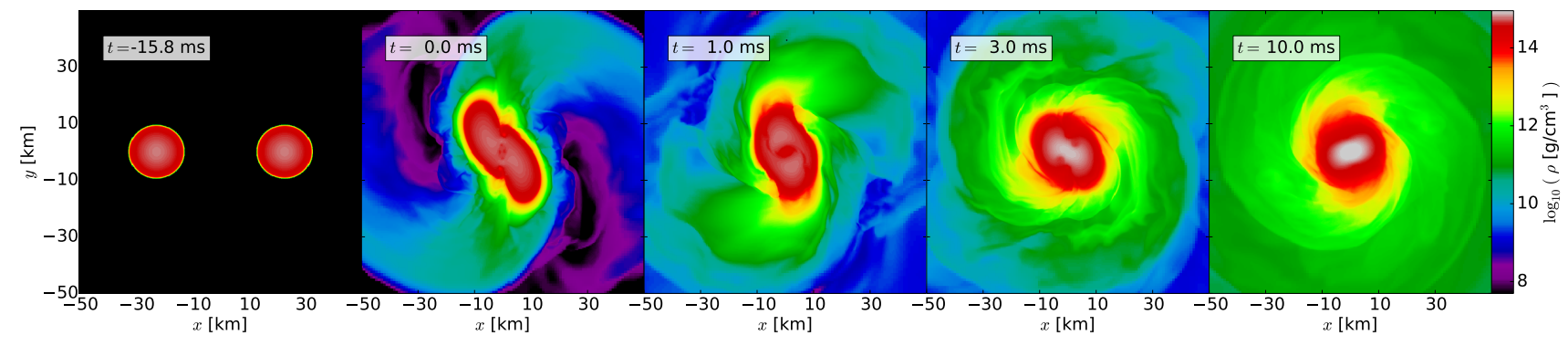

FIG. 8. Snapshots of the rest-mass density on the $(x, y)$ plane for the binary ALF2-q10-M1325. From left to right, the panels refer to five characteristic times: the initial time, the time of the merger, the time right after the merger (i.e., at $t=1.0 \mathrm{~ms}$ ), when the $\ell=m=2$ deformation in the HMNS starts to develop (i.e., at $t=3.0 \mathrm{~ms}$ ), and a later time (i.e., at $t=10.0 \mathrm{~ms}$ ). Note that only in the last panel is the bar-deformed HMNS well defined and quasistationary.

on the $(x, y)$ plane at five characteristic times: the initial time, the time of the merger, the time right after the merger (i.e., at $t=1.0 \mathrm{~ms}$ ), when the stellar core stops oscillating and an $\ell=m=2$ deformation in the HMNS starts to develop (i.e., at $t=3.0 \mathrm{~ms}$ ), and then when the bar-deformed HMNS (cf., region in white) is well defined and with a quasistationary core (i.e., at $t=10.0 \mathrm{~ms}$ ).

Following this phenomenology, it is possible to build a mechanical toy model, whose mathematical details are presented in Appendix A, in which the object produced right after the stellar contact is composed of an axisymmetric disk rotating rapidly at a given angular frequency, say $\Omega(t)$, to which two spheres are connected (e.g., via a shaft) but are also free to oscillate via a spring that connects them (see Fig. 17 in Appendix A). In such a system, the two spheres will either approach each other, decreasing the moment of inertia of the system, or move away from each other, increasing the moment of inertia. Because the total angular momentum is essentially conserved, the system's angular frequency will vary between a minimum value $\Omega_{1}$ (corresponding to the time when the two spheres are at the largest separation) and a maximum value $\Omega_{3}$ (corresponding to the time when the two spheres are at the smallest separation). The values of $\Omega_{1}$ and $\Omega_{3}$ depend nonlinearly on the properties of the system (i.e., the mass and radius of the disk, and the mass of the spheres) but are such that $\Omega_{2}=\frac{1}{2}\left(\Omega_{1}+\Omega_{3}\right)$, just as $f_{2} \approx \frac{1}{2}\left(f_{1}+f_{3}\right)$ in the PSDs we have computed. Stated differently, the mechanical toy model considered here will rotate with an angular frequency that is a function of time and bounded by $\Omega_{1}$ and $\Omega_{3}$. Because the time spent at a given frequency is $\tau_{\Omega} \equiv \Omega /(d \Omega / d t)$, more time is obviously spent at the frequencies $\Omega(t)=\Omega_{1}$ and $\Omega(t)=\Omega_{3}$, where $d \Omega / d t \simeq 0$. As a result, more power is expected to appear in the GW signal at these frequencies, hence producing a low-frequency peak around $\Omega_{1}$ and a high-frequency peak around $\Omega_{3}$. If dissipative processes are present, e.g., if the spring is not ideal and the oscillations are damped, then the angular frequency will tend secularly to $\Omega_{2}$, i.e., $\Omega(t)=\Omega_{2}$ for $t \rightarrow \infty$ (cf., Fig. 18 below). As a result, most of the power in the PSD will appear around $\Omega_{2}$, with two main sidebands at $\Omega_{1}$ and $\Omega_{3}$. Conversely, if dissipative processes are not present, then the GW signal will have contributions at frequencies $\Omega_{2}$ and at its overtones $\Omega_{n} \simeq(n / 2) \Omega_{2}$, such that
$\Omega_{2} \simeq \frac{1}{2}\left(\Omega_{1}+\Omega_{3}\right)$. (Note that in the presence of dissipative processes $\mathrm{a} \simeq$ sign is needed in the estimate of $\Omega_{2}$ because the asymptotic frequency is only approximately the average of $\Omega_{1}$ and $\Omega_{3}$; this is shown in the middle panel of Fig. 18 and reflects the fact that the system is not perfectly balanced.) Overall, and as we will discuss in more detail in Appendix A, this toy model can therefore account for both the presence of the main peak $f_{2}$ and for the two equally distant sidebands at $f_{1}$ and $f_{3}$.

There is a simple way of testing whether these modes are coming just from the immediate post-merger phase or are produced on longer time scales in terms of nonlinear couplings. This is shown in Fig. 9, which reports again the PSDs for the five EOSs and for a representative value of the mass, i.e., $\bar{M}=1.30 M_{\odot}$. Thin solid lines of different colors show the same PSDs as in Fig. 7, with the two vertical dashed lines marking the positions of the peak frequencies $f_{1}$ and $f_{2}$. Shown instead with thick solid lines of the same colors are the PSDs when the waveforms are restricted to the interval $t \in[609,5000] M_{\odot} \approx[3.00,24.63] \mathrm{ms}$, that is, when the first $3 \mathrm{~ms}$ after the merger are cut from the time series. Remarkably, in this case the $f_{1}$ and $f_{3}$ peaks essentially disappear, while the $f_{2}$ peaks remain very strong and without considerable changes in frequency apart for the very soft EOSs. We find this result a convincing validation of the correctness of the toy model and a strong evidence that most of the power in the $f_{1}$ and $f_{3}$ peaks is built essentially over $2-3 \mathrm{~ms}$ after the merger.

We should also note that when the dominant contribution from the initial $f_{1}$ and $f_{3}$ peaks is removed, and hence one is able to measure the power produced by the long evolution of the HMNS, smaller peaks do appear on either side of $f_{2}$, and they are close to the $f_{1}$ or $f_{3}$ frequencies. It is then possible that these smaller-amplitude peaks represent the manifestation of the nonlinear couplings mentioned above and are therefore carriers of interesting information on the properties of the HMNS. Clearly, more work is needed to validate these results and explore the long-term spectrum of the HMNS.

Another concrete indication that the toy model provides a good description of the dynamics right after the merger is offered by Fig. 10, whose top panel shows the full numericalrelativity strain in the + polarization as computed for the bi- 


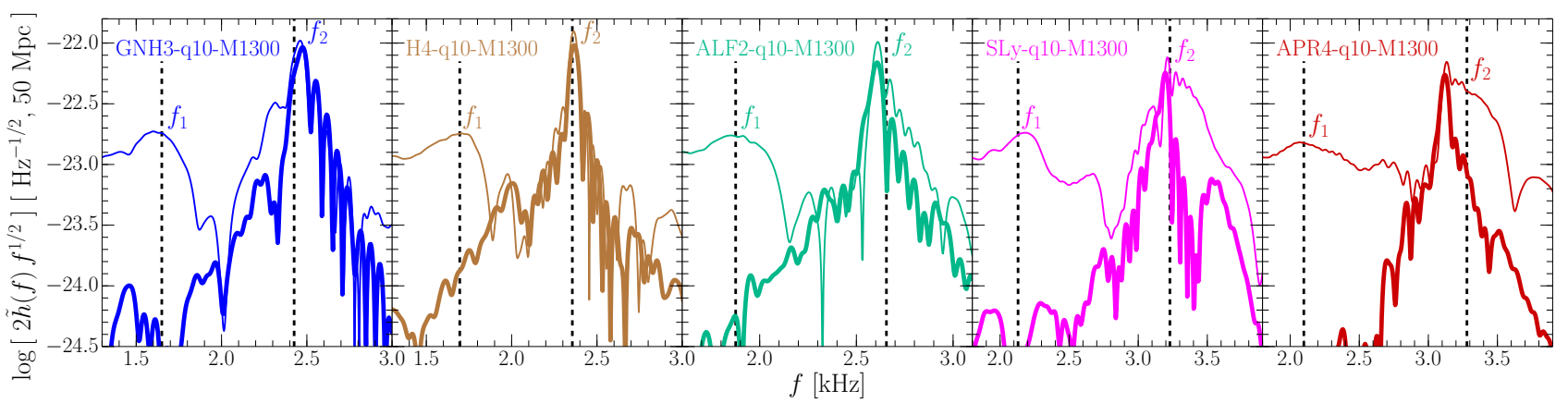

FIG. 9. Selected PSDs $2 \tilde{h}(f) f^{1 / 2}$ relative to binaries with average mass $\bar{M}=1.30 M_{\odot}$. The thin solid lines refer to the PSDs already shown in Fig. 7, while the thick solid lines refer to a time interval excluding the first $3 \mathrm{~ms}$ after the merger, i.e., $t \in[609,5000] M_{\odot} \approx[3.00,24.63] \mathrm{ms}$. The vertical dashed lines indicate the positions of the $f_{1}$ and $f_{2}$ frequencies and highlight that the power in the $f_{1}$ peak is built right after the merger and essentially disappears if the first $3 \mathrm{~ms}$ are excluded.

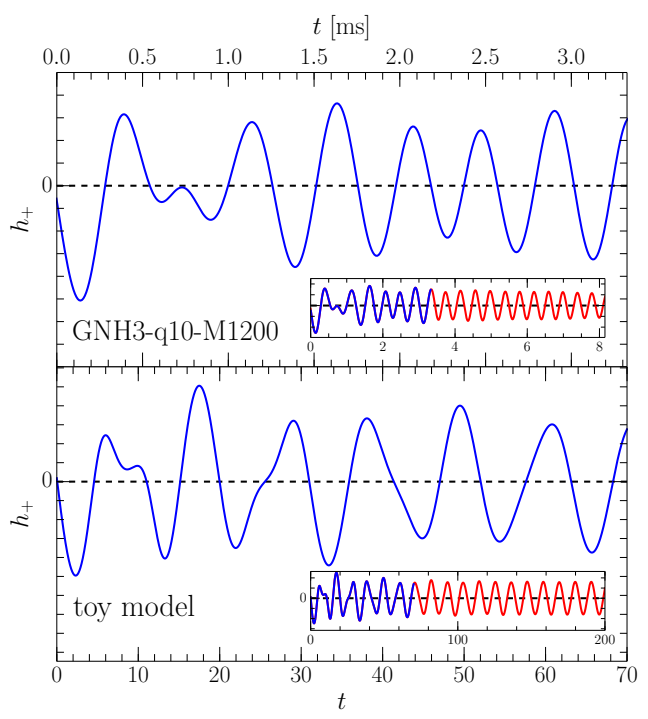

FIG. 10. Top panel: Full numerical-relativity strain in the + polarization computed for the binary GNH3-q10-M1300 for the first $3.3 \mathrm{~ms}$ after the merger. Bottom panel: Strain in the + polarization as computed from the mechanical toy model discussed in Appendix A. The similarities are quite remarkable, especially when considering that the signal shown is representative of the most complex part of the full post-merger signal (cf., insets).

nary $\mathrm{H} 4-\mathrm{q} 10-\mathrm{M} 1300$ for the first $3.3 \mathrm{~ms}$ after the merger. This is to be compared with the GW strain in the + polarization as computed from the mechanical model (see Appendix A for details). The similarities are quite remarkable, especially when considering the crudeness of the toy model and the fact that the signal shown is the most complex one portion of the full post-merger signal, which is far more regular once the HMNS has reached a stationary state (cf., insets). The ability of the toy model to describe the GW emission in the merger phase depends on how well the two stellar cores can be described as two isolated spheres oscillating quasiharmonically. This is obviously a rather good approximation for stiff
EOSs, but the toy model can provide similarly good qualitative agreements essentially for all the waveforms shown in Fig. 6. Hence, at least in principle, the toy model provides a convenient tool to improve the description of the post-merger signal proposed in Ref. [78], possibly opening the way to the fully analytic construction of the post-merger signal for template building.

\section{Correlations of the $f_{\max }$ frequency}

In this section and in the two following it, we will discuss in more detail how to use some of the frequencies in the GW signal discussed above to extract physical information on the stars comprising the binary and hence on their EOS.

We will start by considering the frequency at peak amplitude $f_{\max }$ that, in contrast with the $f_{1}-f_{3}$ frequencies, is an instantaneous frequency and not a feature of the PSDs. We then recall that when restricting the analysis to BNSs having stars with equal masses and set to be $\bar{M}=1.35 M_{\odot}$, Read et al. [31] have recently found that $f_{\max }$ can be effectively characterized only in terms of the tidal deformability $\lambda$ through a relation that is essentially independent of the EOS and was found to be expressed by the relation

$$
\log _{10}\left(\frac{f_{\max }}{\mathrm{Hz}}\right)=3.69652-0.131743 \Lambda^{1 / 5},
$$

where the dimensionless tidal deformability $\Lambda$ is defined as

$$
\Lambda \equiv \frac{\lambda}{\bar{M}^{5}}=\frac{2}{3} \bar{k}_{2}\left(\frac{\bar{R}}{\bar{M}}\right)^{5} \text {, }
$$

and $\bar{k}_{2}$ is the $\ell=2$ dimensionless tidal Love number. [The empirical relation (22) is shown as a dotted line in Fig. 11.] Already in Ref. [31] it was noted that this behavior is reminiscent of the so-called universal "I-Love-Q" relations [79, 80] that have been pointed out for isolated neutron stars and have since been the subject of intense research [81-93]. A similar behavior has been found also within an effective-one-body 


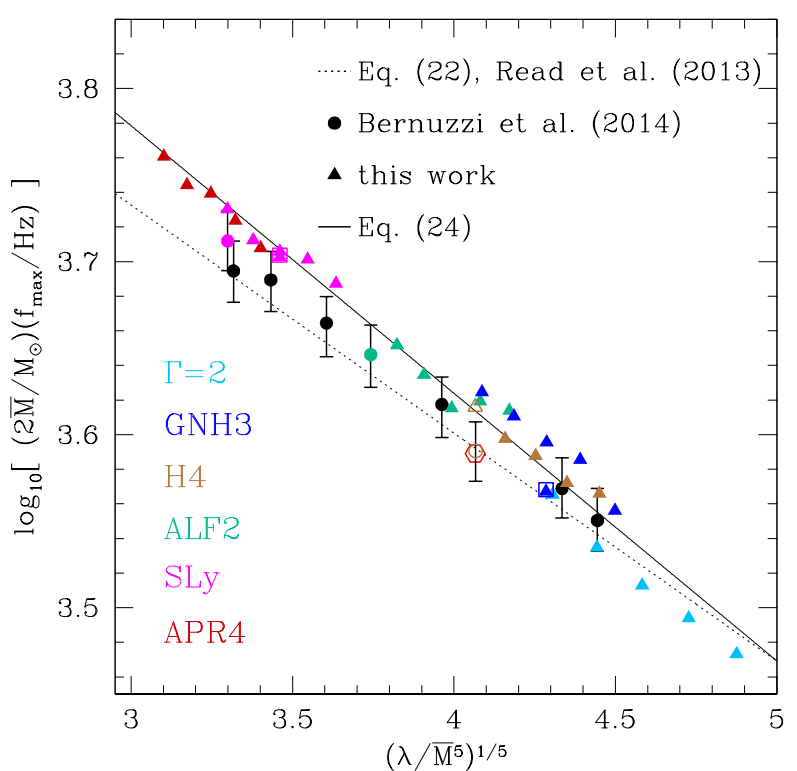

FIG. 11. Mass-weighted frequencies at amplitude maximum $f_{\max }$ shown as a function of the dimensionless tidal deformability $\lambda / \bar{M}^{5}$. Filled circles refer to the data from [32], while colored triangles indicate the data from our binaries (triangles in boxes refer to the unequal-mass binaries GNH3-q09-M1300 and SLy-q09-M1300). The dotted line shows the relation suggested in [31] [i.e., Eq. (22)], while the solid line represents the best fit to our data [i.e., Eq. (24)]. Note that the systematic differences between circles and triangles are due to the small differences in the definition of the time of merger; indeed the red hexagon represents the new position to which the value for $\bar{M} f_{\max }$ (empty brown triangle) moves to when the difference in the time of merger is taken into account and is very close to the one in Ref. [32] (empty brown circle).

(EOB) description of the tidal effects in BNSs [32]. More specifically, the EOB analytic approach has revealed quasiuniversal relations of the mass-rescaled GW frequency and of the binding energy at the time of merger when expressed as functions of $\ell=2$ dimensionless tidal coupling constant $\kappa_{2}^{T}$, which is related to tidal deformability by $\lambda=16 \kappa_{2}^{T} \bar{M}^{5} / 3$ in an equal-mass case. Together with semianalytic calculations, Ref. [32] also reported the results of numerical-relativity simulations of equal-mass BNSs for nine different EOSs finding both a good match with the results of [31] and with those of the EOB approximation (the data relative to the numerical simulations in Ref. [32] are shown with filled circles in Fig. 11).

Because the numerical-relativity results both of Ref. [31] and of Ref. [32] were restricted to the analysis of equal-mass systems with average mass $\bar{M}=1.35 M_{\odot}$, it is interesting to reconsider these quasiuniversal relations when also the mass of the system is allowed to vary. Of course this was already explored in [32] within the EOB approximation, but we can now extend it also to a fully nonlinear regime.

A summary of the correlation of the $f_{\max }$ frequency with the tidal deformability is shown in Fig. 11, where we indicate with a dotted line the empirical relation suggested in [31] [i.e., Eq. (22)], with filled circles the data from [32], and with colored triangles the data from our binaries (triangles in boxes refer to the unequal-mass binaries GNH3-q09-M1300 and $\mathrm{SLy}-\mathrm{q} 09-\mathrm{M} 1300$ ). Clearly, also the results presented here support the universality of $f_{\max }$ as a function of the dimensionless tidal deformability $\Lambda$. At the same time, since expression (22) does not have any information about the mass of the system $\bar{M}$, this can be introduced rather simply through the expression

$$
\log _{10}\left(\frac{f_{\max }}{\mathrm{Hz}}\right) \approx 4.2423-0.1546 \Lambda^{1 / 5}-\log _{10}\left(\frac{2 \bar{M}}{M_{\odot}}\right) .
$$

Stated differently, the universality is really in the quantity $\bar{M} f_{\max }$, as already pointed out in Ref. [32]. Expression (24) is shown as a solid line in Fig. 11 and provides a very good fit of the data, with a maximum relative difference between the simulations and fitted values that is only $\sim 0.7 \%$.

Before concluding this section we should remark about the amount of scattering in the numerical data. There could be a number of reasons behind this scatter (e.g., different codes, different initial data, etc.) but we believe that the largest source of difference is systematic and comes from a slightly different definition of the merger time. For example, while we define it to be the time of the first maximum in the $\ell=m=2$ mode amplitude $|h|=\left(h_{+}{ }^{2}+h_{\times}{ }^{2}\right)^{1 / 2}$, Ref. [31] (and possibly Ref. [32]) defined it as the time with the maximum amplitude of $\left|h_{+}\right|$. Indeed, if we adopt the same definition as in $[31,32]$, then the systematic difference disappears. This is illustrated with the binary $\mathrm{H} 4-\mathrm{q} 10-\mathrm{M} 1350$, for which we use the same initial data as in Ref. [32]; the red hexagon represents the new position to which the value for $\bar{M} f_{\max }$ (empty brown triangle) moves when the difference in the time of merger is taken into account and clearly the new value corresponds closely to the one in Ref. [32] (empty brown circle).

\section{Correlations of the $f_{1}$ frequency}

As already discussed in Ref. [30], possibly one of the most interesting aspects of our spectral analysis is that there is a very clear correlation between the low-frequency peak $f_{1}$ and the stellar compactness $\mathcal{C} \equiv \bar{M} / \bar{R}$. This is shown in Fig. 12, which reports the values of the $f_{1}$ frequencies plotted as a function of $\mathcal{C}$ for the various EOSs. Each cross refers to a given mass and the frequencies grow with mass; i.e., for each EOS the smallest $f_{1}$ frequency corresponds to the smallest mass and the largest frequency to the largest mass. Also shown as a shaded grey band is the estimate of the total error, which is effectively dominated by the fitting procedure of the PSD, since the average numerical error from the simulations is estimated to be $0.06 \mathrm{kHz}$, while the average uncertainty in the fitting procedure of the PSD is of $0.2 \mathrm{kHz}$ (see also Ref. [70]).

The behavior of the low-frequency peak is remarkably con- 


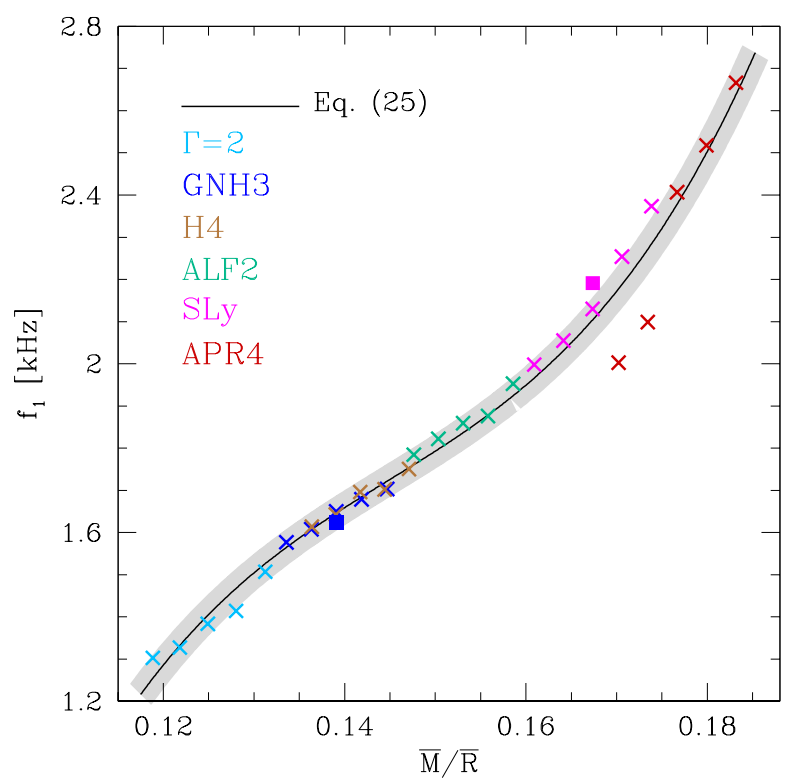

FIG. 12. Values of the low-frequency peaks $f_{1}$ shown as a function of the stellar compactness for the six different EOSs considered; note the universal behavior exhibited also by unequal-mass binaries (filled squares). Shown as a solid black line is the cubic fit, while the grey band is the estimate of the total errors (cf., left panel of Fig. 2 of [30]).

sistent with a simple cubic polynomial of the type

$$
\frac{f_{1}}{\mathrm{kHz}} \approx a_{0}+a_{1} \mathcal{C}+a_{2} \mathcal{C}^{2}+a_{3} \mathcal{C}^{3}
$$

where

$$
\begin{aligned}
& a_{0}=-22.0717 \pm 6.477, \quad a_{1}=466.616 \pm 131.2, \\
& a_{2}=-3131.63 \pm 878.7, \quad a_{3}=7210.01 \pm 1947 .
\end{aligned}
$$

The fit yields then a $\chi$-squared of 0.09 , with a fitting uncertainty $\lesssim 0.06 \mathrm{kHz}$ and a maximum difference between data from the simulations and the polynomial function that is $\sim 0.17 \mathrm{kHz}$. The very tight fit in Fig. 12 is somewhat spoiled by the behavior of two binaries with the APR4 EOS, namely, APR4-q10-M1275 and APR4-q10-M1300. The reason behind the large scatter in these two models is rather simple and is due to the fact that for these binaries the power in the $f_{1}$ peak is very small because the EOS is very soft (cf., Fig. 7). Stated differently, the softness of the EOS and the smallness of the mass in these binary prevent the large bounce of the stellar cores after the first collision. As a result, the GW emission at low frequencies is considerably reduced. Excluding these two points from our data set of the $f_{1}$ frequencies would reduce the maximum difference from the fitting expression (25) to be only $\sim 0.09 \mathrm{kHz}$.

We should note that the $f_{\max }$ and $f_{1}$ frequencies are clearly distinct, both numerically (the actual values in $\mathrm{Hz}$ are different) and physically (while the first is an instantaneous fre-

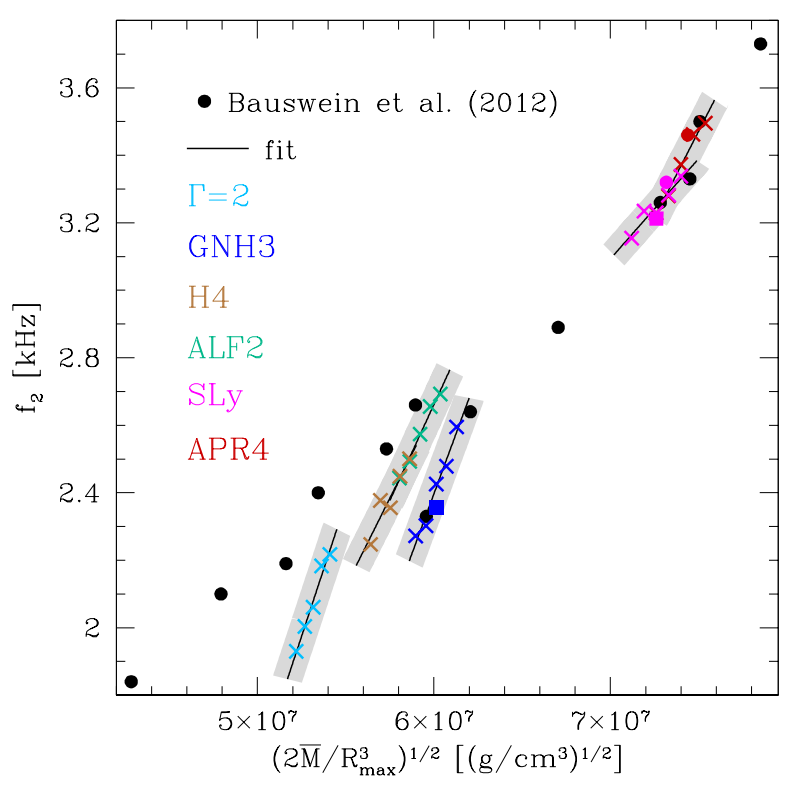

FIG. 13. Values of the $f_{2}$ peak frequencies shown as a function of the pseudoaverage rest-mass density $\left(2 \bar{M} / R_{\max }^{3}\right)^{1 / 2}$, where colored crosses and squares refer to equal- and unequal-mass binaries, respectively. Shown as solid black lines are the linear fits within each EOS, while the grey band is the estimate of the total errors. We also report as filled circles the results presented in Ref. [25] for $\bar{M}=1.35 M_{\odot}$ binaries, where we use magenta and red circles for the binaries that correspond to our models SLy-q10-M1350 and APR4-q10-M1350, respectively.

quency, the second is a feature of the PSD). Yet, these frequencies tend to blend in the limit of very soft EOSs and small masses. For example, the values of $f_{\max }$ and $f_{1}$ have a difference of $\lesssim 0.3 \%$ and $\lesssim 0.07 \%$ for the binaries APR4-q10-M1275 and APR4-q10-M1300, respectively.

A concluding remark: we have shown that also the $f_{1}$ frequency exhibits a quasiuniversal behavior with the stellar compactness, and hence that, together with the $f_{\max }$ frequency, it can be added to the increasingly large set of stellar properties that have an EOS-independent behavior. Yet, this does not add much to our understanding of why such a behavior should take place in nature. Two papers have recently proposed interesting explanations for this behavior, suggesting that universality arises as an emergent approximate symmetry [92], or because of an almost incompressible behavior of compact stars at high densities [93]. Interestingly, whatever leads to the $f_{1}$ universal behavior cannot be attributed to an emergent symmetry (which is definitely lost at the merger) or to an incompressible behavior (large shocks are produced at the merger). Rather, it suggests that the universal behavior is preserved also in the absence of symmetries or when large compressions take place. 


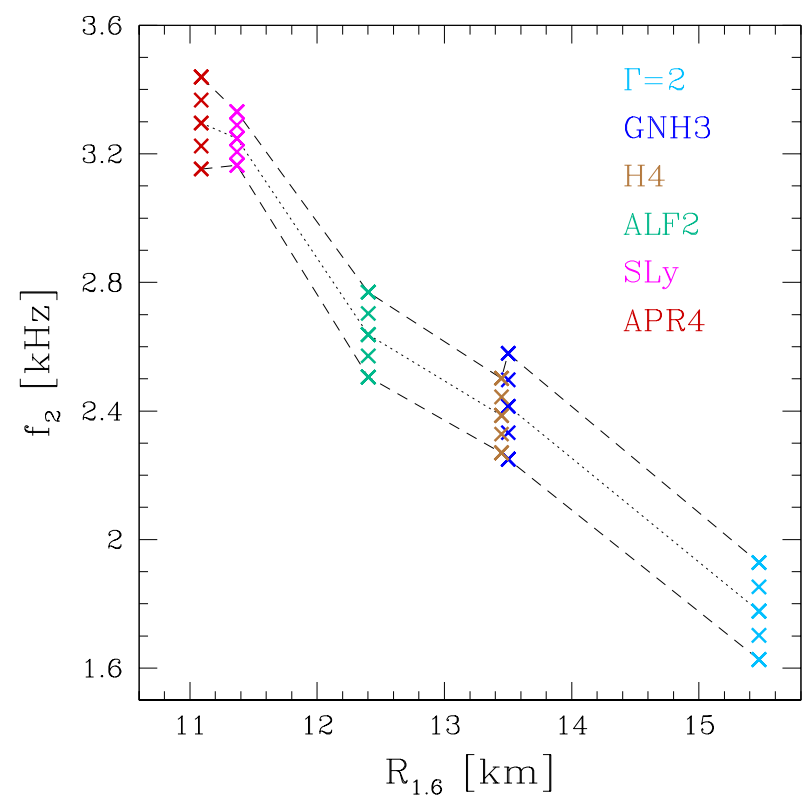

FIG. 14. Values of the $f_{2}$ peak frequencies shown in terms of $R_{1.6}$, the radius of a nonrotating NS with $\bar{M}=1.6 M_{\odot}$. The various crosses refer to the $f_{2}$ frequencies at masses $\bar{M} / M_{\odot}=$ $1.250,1.275,1.300,1.325$, and 1.350 ; these values are either available directly, or interpolated from the mass values used in the simulations. The dashed and dotted lines connect, respectively, the largest, the smallest, and the intermediate masses, showing that it is difficult to find a bilinear fitting across different EOSs.

\section{E. Correlations of the $f_{2}$ frequency}

Historically the first spectral feature to have been detected and discussed [8, 23-25], the high-frequency $f_{2}$ peak has been shown to correlate with a number of physical quantities of the progenitor stars. The most representative example is shown in Fig. 13, which reports the value of the $f_{2}$ frequency as a function of the pseudoaverage rest-mass density $\left(2 \bar{M} / R_{\max }^{3}\right)^{1 / 2}$ for the different EOSs, where $R_{\max }$ is the radius of the maximum-mass nonrotating configuration for the EOS under consideration. Indicated with crosses and filled squares are the equal- and unequal-mass binaries, respectively. Also reported as solid circles are the results presented in Fig. 4 of Ref. [25] and relative to $\bar{M}=1.35 M_{\odot}$ binaries, where we have indicated with magenta and red circles those models that have the same EOS considered here (i.e., SLy-q10-M1350 and APR 4-q10-M1350, respectively). Overall, the mass dependence in $f_{2}$ (i.e., what distinguishes different points of the same color) does not suggest a tight universal correlation in our data, although one may conclude differently if only binaries of one mass were considered as in Ref. [25]. The situation does not improve if instead of using the fitted value for $f_{2}$ following the algorithm in Sec. III B, we simply use the local maximum value of the high-frequency peak. However, within each EOS, the data are rather well reproduced with a linear fit (the $\chi$-squared value is $\lesssim 0.004$ ). Although EOS is dependent, these fits still provide a set of relations that can be used to constrain the EOS, as we anticipated in [30] and will further discuss in Sec. V G.

Finally, shown in Fig. 14 are the values of the $f_{2}$ peak frequencies in terms of $R_{1.6}$, that is, the radius of a nonrotating NS with $\bar{M}=1.6 M_{\odot}$ (cf., Fig. 23 of Ref. [26]). Because it is relevant here to correlate frequencies with the same mass, the various crosses refer to the $f_{2}$ frequencies at masses $\bar{M} / M_{\odot}=1.250,1.275,1.300,1.325$, and 1.350 ; these values are either available directly, or interpolated from the mass values used in the simulations (see Table II). As it is apparent after connecting the different crosses with the same mass, e.g., with the dashed and dotted lines that connect, respectively, the largest, the smallest, and the intermediate masses, it is difficult to find a bilinear fitting function across the different EOSs as the one suggested by Eq. (3) of Ref. [26].

\section{F. Other potential correlations}

In addition to the correlations of $f_{\max }, f_{1}$, and $f_{2}$ discussed, respectively, in Secs. V C, VD, and VE, we have explored whether other empirical correlations can be found between these frequencies and physical quantities of the binary system, e.g., the compactness $\bar{M} / \bar{R}$, the average density $\left(\bar{M} / \bar{R}^{3}\right)^{1 / 2}$, the pseudoaverage rest-mass density $\left(\bar{M} / R_{\max }^{3}\right)^{1 / 2}$, or the dimensionless tidal deformability $\left(\lambda / \bar{M}^{5}\right)^{1 / 5}$.

The results of this exploration are collected in Fig. 15, where the left panel refers to the absolute values of the frequencies in $\mathrm{kHz}$, e.g., $f_{\max }$, while the right one refers to the corresponding normalized expressions, e.g., $\bar{M} f_{\max }$. As in the previous figures, the different EOSs are distinguished with different colors and the various symbols within each EOS refer to binaries with different masses; quite generally, the highest value of the frequency is the one corresponding to the largest mass in the sequence of the same EOS.

Note that when restricted to a single binary mass, the correlation of the $f_{2}$ frequency with the average rest-mass density appears to be much tighter than it first appeared [25], indicating that it is essential to consider, in addition to a large set of EOSs, also a wide range of masses (and mass ratios). It is also interesting to note that the correlations can become "tighter" depending on what is the normalization used. In other words, the "universality" of the max-amplitude frequency with the average compactness $\mathcal{C}$ is different whether one is considering $f_{\max }$ (left panel of Fig. 15) or $\bar{M} f_{\max }$ (right panel of Fig. 15). Conversely, a larger spread is seen in the relation of the low-frequency peak with compactness if $\bar{M} f_{1}$ is used instead of $f_{1}$. We do not have a simple explanation for this behavior, which, however, has been observed repeatedly within the framework of quasiuniversal relations (see discussions in Refs. [82] and [85]).

Finally, the general overview of Fig. 15 allows us to appreciate the presence of at least two new "quasiuniversal" correlations in addition to the ones already discussed [i.e., $\bar{M} f_{\max }$ vs. $\left(\lambda / \bar{M}^{5}\right)^{1 / 5}$ and $f_{1}$ vs $\left.\bar{M} / \bar{R}\right]$. Particularly interesting are the correlation of $f_{\max }$ with the average density $\left(\bar{M} / \bar{R}^{3}\right)^{1 / 2}$ 

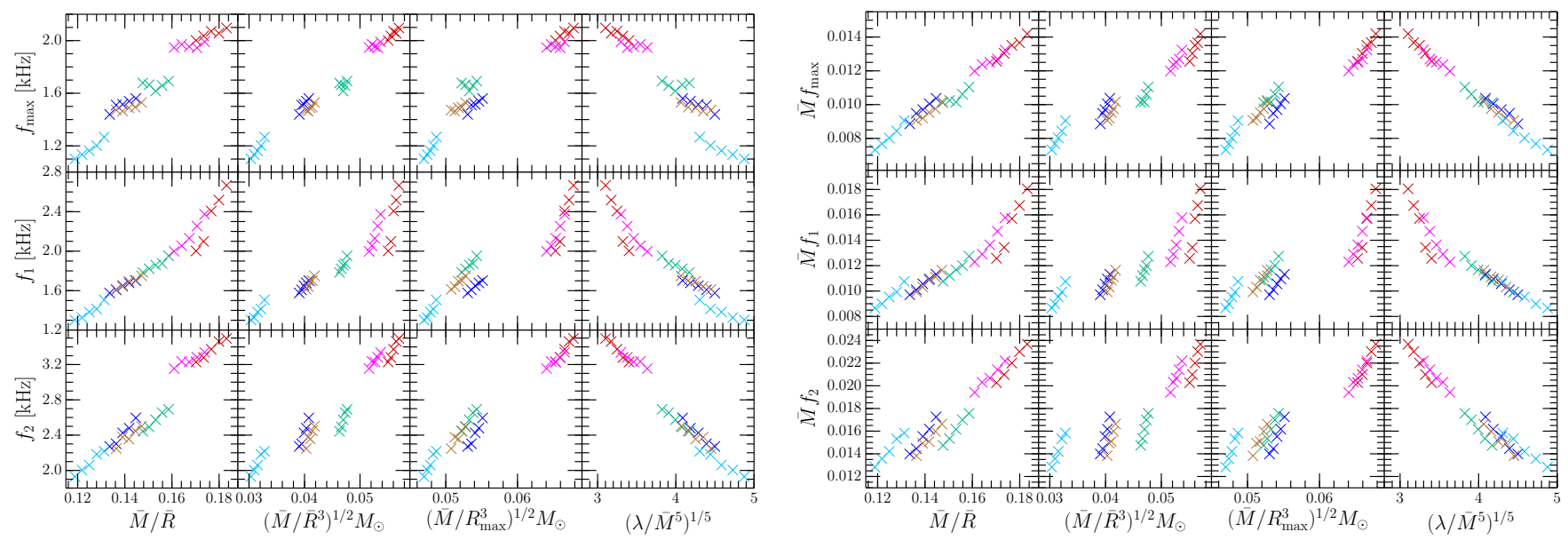

FIG. 15. Other empirical correlations between the $f_{\max }, f_{1}$, and $f_{2}$ frequencies and the physical quantities of the binary system, e.g., the compactness $\bar{M} / \bar{R}$, the average density $\left(\bar{M} / \bar{R}^{3}\right)^{1 / 2}$, the pseudoaverage rest-mass density $\left(\bar{M} / R_{\max }^{3}\right)^{1 / 2}$, or the dimensionless tidal deformability $\left(\lambda / \bar{M}^{5}\right)^{1 / 5}$. The left panel refers to frequencies expressed in $\mathrm{kHz}$, while the right one refers to dimensionless frequencies expressed in terms of the average mass $\bar{M}$.

(right column of the left panel), but also the correlations of $f_{1}$ and $f_{2}$ with the dimensionless tidal deformability $\left(\lambda / \bar{M}^{5}\right)^{1 / 5}$ (left column of the left panel). These correlations appear also when expressing the results in terms of the corresponding normalized frequencies $\bar{M} f_{\max }, \bar{M} f_{1}$, and $\bar{M} f_{2}$ (right panel), although the scattering can also increase in this case. In the following Sec. VG we will discuss how to translate two of these correlations into potential measurements of the EOS if a post-merger signal with sufficiently large SNR is detected. At the same time, Fig. 15 highlights that much more information can be extracted from these frequencies, and hence more work is needed to fully exploit the spectral information on the post-merger GW signal from BNSs.

\section{G. Constraining the EOS}

After having discussed in detail the spectral features of the post-merger signal, some considerations should now be made on a new approach that uses the correlations between the PSDs and the stellar properties to set tight constraints on the EOS of nuclear matter. This approach has already been presented in some detail in Ref. [30], but we recall it here for completeness.

Essential for the success of this approach is a "clear identification" of the peaks in the PSD of the post-merger signal, which, in turn, will require a high SNR and will depend on the EOS. More concretely, a measure by Advanced LIGO of the $f_{1}$ peak with a $\mathrm{SNR}=5$ will require a binary at a distance of about $25 \mathrm{Mpc}$ for the APR4 EOS and of about $40 \mathrm{Mpc}$ for the (GNH3) EOSs. On the other hand, larger distances of, respectively, about 50 or $115 \mathrm{Mpc}$ can be afforded to achieve a measurement of the $f_{2}$ peak with a SNR $=5$. These distances are clearly smaller than the typical horizon scales of BNSs expected for the advanced detectors, limiting the probability of detection of such events. Yet, as we discuss below, the mea- surement would be so significant that a single detection would be sufficient to constrain the EOS, at least in principle.

Let us therefore assume that the GW signal from a BNS has been detected and that the source is sufficiently close that all of the spectral features are clearly identifiable. Using the measured values of $f_{1}$ and $f_{2}$ we can draw a series of curves in the $(\bar{M}, \bar{R})$ plane. The first of these curves will be the one expressing the relation $\bar{M}=\bar{M}\left(\bar{R}, f_{1}\right)$ (solid grey line), and, as discussed in Sec. VD, this line will be the same for all EOSs. At the same time, the measurement of $f_{2}$ will lead to as many lines as the EOSs that are considered, where each curve will express the relation $\bar{M}=\bar{M}\left(\bar{R}, f_{2} ;\right.$ EOS) (solid colored lines). The resulting set is shown in Fig. 16, where the leftmost panel refers to the GNH3 EOS, while the rightmost one refers to the APR4 EOS. All panels are specific to a binary with $\bar{M}=1.30 M_{\odot}$, but essentially identical plots can be shown also for other masses, and we limit to one mass only for compactness. The uncertainties, both theoretical and experimental, in the measurement of $f_{1}$ and $f_{2}$ make the correlation curves $\bar{M}=\bar{M}\left(\bar{R}, f_{1}\right)$ and $\bar{M}=\bar{M}\left(\bar{R}, f_{2} ;\right.$ EOS $)$ be effectively "bands", rather than thin lines, each with its own width representing the corresponding probability distribution.

Concentrating, for example, on the left panel, which refers to the binary GNH3-q10-M1300, we can see that the $\bar{M}=$ $\bar{M}\left(\bar{R}, f_{1}\right)$ relation intersects each of the various equilibrium curves (colored dashed lines) at one point (e.g., at $\bar{M} \simeq$ $1.3 M_{\odot}, \bar{R} \simeq 13.8 \mathrm{~km}$ for the GNH3 EOS), but also that other "crossings" take place for the other EOSs. However, when using also the relations $\bar{M}=\bar{M}\left(\bar{R}, f_{2} ;\right.$ EOS $)$, some EOSs can be readily excluded (e.g., APR4, ALF2, and GNH3, in this example) and only the H4 and GNH3 EOSs have crossings (or "near crossings") between the equilibrium-models curves and the frequency-correlations curves. A more careful Bayesian analysis can associate with each of these near crossings a certain probability depending on the width of the different bands, 


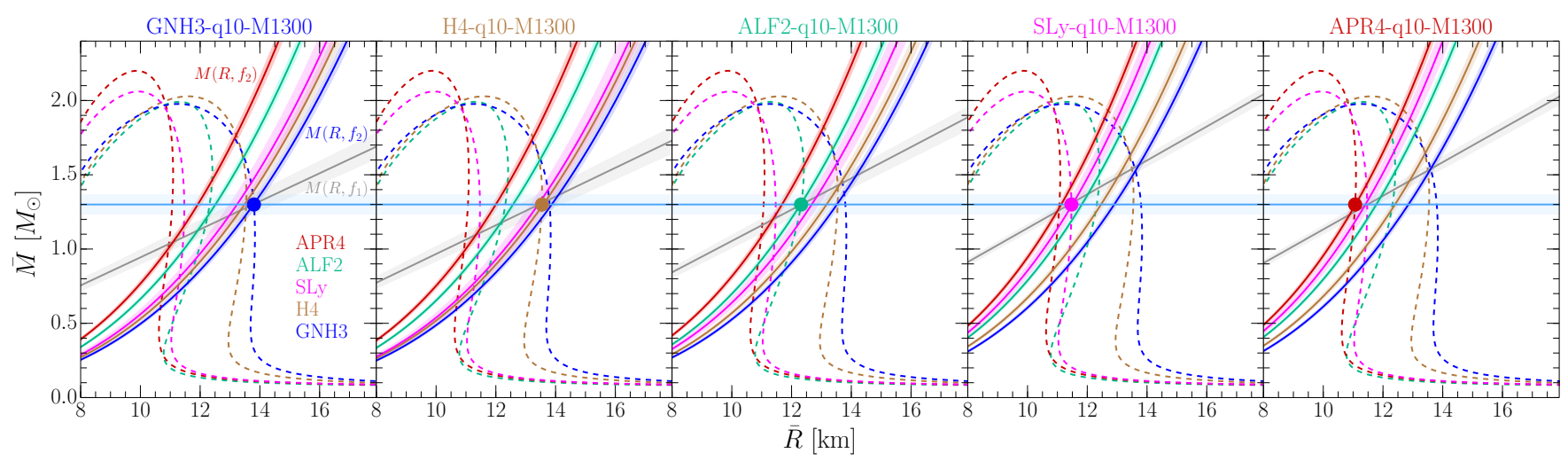

FIG. 16. Examples of use of the spectral features to constrain the EOS. Once a detection is made, the relations $\bar{M}=\bar{M}\left(\bar{R}, f_{1}\right)$ and $\bar{M}=\bar{M}\left(\bar{R}, f_{2} ;\right.$ EOS $)$ (colored solid lines) will cross at one point the curves of equilibrium configurations (colored dashed lines). Knowledge of the mass of the system (horizontal line) will provide a fourth constraint, removing possible degeneracies. The leftmost panel refers to the GNH3 EOS, while the rightmost one refers to the APR4 EOS. All panels are specific to a binary with $1.3 M_{\odot}$, but essentially identical plots can be shown also for other masses and we limit ourselves to a single mass only for compactness.

thus providing additional support (at least from a statistical point of view) to one single EOS over the others; the results of this analysis will be presented in a forthcoming paper [94].

The ability to single out the correct EOS over the others is significantly increased if the mass of the binary is known from the inspiral signal ${ }^{5}$. In this way, an additional band in the $(\bar{M}, \bar{R})$ plane, shown as a horizontal light-blue stripe in the panel, can be drawn. This stripe, whose width we have taken to correspond to an error of $10 \%$ in the determination of the mass, will intersect the three bands relative to the GNH3 EOS in a small patch (ideally at one point only if there were no errors in the measures of the frequencies; cf., blue solid circle), while this will not be the case for the other crossings. Hence, the simultaneous fulfilling of four distinct constraints [i.e., of four crossings at a point in the $(\bar{M}, \bar{R})$ plane] represents a new and effective approach to constrain the EOS of nuclear matter.

At least in principle, this tool is so effective that a single detection with sufficiently high SNR would be able to constrain the EOS. In practice, even a high-SNR detection would be able to mark only one point in the $(\bar{M}, \bar{R})$ plane, which may well not be covered by any of the presently known nuclearphysics EOSs. Furthermore, the detection would only set a confidence level, possibly very high, on the probability that the correct EOS should pass over that point in the $(\bar{M}, \bar{R})$ plane. Hence, it is clear that the success of this approach relies on the ability of making a sufficient number of detections with small theoretical and experimental uncertainties in the measure of the peak frequencies. In this respect, the approach presented here will set constraints on the EOS only in a statistical sense. Unfortunately, as we will discuss in Sec. V H, only a small fraction of the expected detection rate of $\sim 40 \mathrm{yr}^{-1}[4]$

\footnotetext{
${ }^{5}$ We note that in practice it will be difficult to distinguish the mass ratio from the chirp signal and hence the total mass of the system will represent a first reasonable approximation.
}

will be at sufficiently close distances.

Before closing this section we will make some additional considerations. First, even if the measurement of the mass is not available from the inspiral (this is possible, though not likely as the chirp mass is one of the most robust measurements), the degeneracies in the EOSs could be removed with a few positive detections, which would tend to favor one EOS over the others. Second, even if none of the presently known EOSs is the correct one to describe the properties of nuclear matter, a number of detections at different masses would be able to actually "trace" the curve of equilibrium models in the $(\bar{M}, \bar{R})$ plane; this track would then need to be reproduced by any microphysical description of the EOS. Third, if only the $f_{2}$ frequency is measurable, the approach discussed above can still be used as long as the mass is known; in this case three and not four curves will have to cross at one point. Fourth, most of our simulations refer to equal-mass binaries, but we expect that the $f_{1}$ and $f_{2}$ frequencies will not be very sensitive to the initial mass ratio; this was already shown by Refs. [25, 27] and is confirmed by the two unequal-mass binaries that are part of our sample. Fifth, realistic values of the stellar spins are not likely to influence the frequencies significantly given that the largest contribution to the angular momentum of the HMNS comes from the orbital angular momentum and not from the initial spins of the stars $[50,95]$. Finally, because the $f_{1}$ peak is produced soon after the merger, it should not be affected significantly by magnetic fields and radiative effects, whose modifications emerge on much larger time scales $[14,96]$.

\section{H. Detectability of the peak frequencies}

Obviously, our method can work only if the frequencies of the peaks can be measured in GW detectors, there is a sufficient number of detections, and the uncertainties in the measure of the frequencies are sufficiently small. As shown 


\begin{tabular}{l|c|c|c|c|c|c|c}
\hline \hline Binary & $f_{\max }$ & $f_{1}$ & $f_{2}$ & SNR & SNR $_{\text {cont }}$ & SNR $_{f_{1}}$ & SNR $_{f_{2}}$ \\
& {$[\mathrm{~Hz}]$} & {$[\mathrm{Hz}]$} & {$[\mathrm{Hz}]$} & & & & \\
\hline GAM2-q10-M1350 & 1101 & 1303 & 1930 & 5.2 & 2.7 & 5 & 6 \\
GAM2-q10-M1375 & 1134 & 1327 & 2004 & 5.2 & 2.4 & 6 & 5 \\
GAM2-q10-M1400 & 1163 & 1384 & 2061 & 5.2 & 2.3 & 5 & 5 \\
GAM2-q10-M1425 & 1202 & 1414 & 2183 & 5.4 & 2.4 & 6 & 5 \\
GAM2-q10-M1450 & 1267 & 1507 & 2127 & 5.5 & 2.3 & 5 & 5 \\
\hline GNH3-q10-M1250 & 1439 & 1577 & 2272 & 4.5 & 2.1 & 4 & 14 \\
GNH3-q10-M1275 & 1510 & 1608 & 2302 & 4.6 & 2.1 & 4 & 15 \\
GNH3-q10-M1300 & 1515 & 1650 & 2425 & 4.8 & 2.2 & 3 & 14 \\
GNH3-q10-M1325 & 1539 & 1679 & 2479 & 5.0 & 2.3 & 4 & 14 \\
GNH3-q10-M1350 & 1560 & 1703 & 2595 & 5.0 & 2.2 & 4 & 10 \\
GNH3-q09-M1300 & 1420 & 1624 & 2355 & 5.0 & 2.3 & 5 & 16 \\
\hline ALF2-q10-M1225 & 1678 & 1785 & 2443 & 4.2 & 1.6 & 3 & 7 \\
ALF2-q10-M1250 & 1664 & 1822 & 2493 & 4.3 & 1.5 & 3 & 5 \\
ALF2-q10-M1275 & 1617 & 1859 & 2574 & 4.4 & 1.6 & 3 & 7 \\
ALF2-q10-M1300 & 1658 & 1876 & 2655 & 4.6 & 1.7 & 3 & 5 \\
ALF2-q10-M1325 & 1692 & 1953 & 2693 & 4.7 & 1.7 & 3 & 3 \\
\hline H4-q10-M1250 & 1473 & 1613 & 2247 & 4.5 & 2.0 & 4 & 9 \\
H4-q10-M1275 & 1464 & 1643 & 2377 & 4.6 & 2.0 & 3 & 11 \\
H4-q10-M1300 & 1489 & 1696 & 2356 & 4.8 & 2.1 & 4 & 12 \\
H4-q10-M1325 & 1494 & 1702 & 2449 & 4.9 & 2.2 & 4 & 13 \\
H4-q10-M1350 & 1529 & 1751 & 2501 & 5.0 & 2.3 & 3 & 14 \\
\hline SLy-q10-M1250 & 1947 & 1998 & 3154 & 4.3 & 1.4 & 2 & 6 \\
SLy-q10-M1275 & 1971 & 2055 & 3235 & 4.4 & 1.5 & 3 & 6 \\
SLy-q10-M1300 & 1954 & 2130 & 3229 & 4.6 & 1.4 & 3 & 9 \\
SLy-q10-M1325 & 1946 & 2254 & 3282 & 4.7 & 1.5 & 3 & 7 \\
SLy-q10-M1350 & 1991 & 2373 & 3338 & 4.8 & 1.5 & 3 & 9 \\
SLy-q09-M1300 & 1940 & 2191 & 3212 & 4.6 & 1.6 & 3 & 8 \\
\hline APR4-q10-M1275 & 2001 & 2003 & 3229 & 4.3 & 1.3 & 2 & 5 \\
APR4-q10-M1300 & 2035 & 2099 & 3279 & 4.6 & 1.4 & 4 & 4 \\
APR4-q10-M1325 & 2071 & 2407 & 3373 & 4.6 & 1.3 & 3 & 4 \\
APR4-q10-M1350 & 2056 & 2518 & 3462 & 4.7 & 1.3 & 3 & 3 \\
APR4-q10-M1375 & 2096 & 1953 & 2693 & 4.9 & 1.3 & 2 & 3 \\
\hline \hline & & & & & \\
\hline
\end{tabular}

TABLE III. Values of the $f_{\max }, f_{1}$, and $f_{2}$ peak frequencies, where the last two are computed with the fitting procedure. For all waveforms, the SNR is computed from $7.4 \mathrm{~ms}$ before the merger and up to $24.6 \mathrm{~ms}$ after the merger, and $\mathrm{SNR}_{\text {cont }}$ is the value computed for the waveform as above but with $f \geq f_{\text {cont }}$. The last two columns report rounded to the integer figure the "effective SNR" at the frequencies $f_{1}$ and $f_{2}$, that is, the ratio between the PSD of the signal and PSD of the detector noise at those frequencies. Note that for the effective SNR the values differ at most of a factor of 3 and are even comparable for some binaries. For all cases, we assumed GW detections for sources with optimal orientation at $50 \mathrm{Mpc}$ and the noise spectrum of Advanced LIGO.

qualitatively in Fig. 7, this is possible for sufficiently close events. For more quantitative estimates, we have computed and reported in Tables III and IV the SNRs and the error estimates for the models we simulated. In particular, Table III lists, in addition to the peak frequencies $f_{1}$, and $f_{2}$, the SNR computed from each waveform in the time interval starting at $7.4 \mathrm{~ms}$ before the merger and ending at $24.6 \mathrm{~ms}$ after the merger. The choice of a fixed time interval was made to facilitate the comparison of the detectability across different models. For most models, this interval includes most of the postmerger signal, but only a few orbits of the inspiral.

The sixth column of Table III shows $\mathrm{SNR}_{\text {cont }}$, that is, the SNR computed using the waveforms in the same interval as above, but by considering only frequencies larger than the contact frequency $f_{\text {cont }}$, in order to single out the contribution to the detected GW signal of the post-merger phase. In this respect, $\mathrm{SNR}-\mathrm{SNR}_{\text {cont }}$ represents the SNR of the (short) inspiral only and can be seen as a lower limit on the detectability of these signals. The last two columns list the "effective SNR" at the peak frequencies $f_{1}$, and $f_{2}$, that is, the ratio between the PSD of the signal and the PSD of the detector at those frequencies, i.e., $\mathrm{SNR}_{f_{1,2}} \equiv 2 \tilde{h}\left(f_{1,2}\right) \sqrt{f_{1,2}} / S_{h}\left(f_{1,2}\right)$. Note that a smaller power in the $f_{1}$ peak is compensated by a smaller detector noise at lower frequencies, so that the SNRs at the frequencies $f_{1}$ and $f_{2}$ differ at most by a factor of 3 and are even comparable for some binaries. For all cases, we have assumed GW detections for sources with optimal orientation at $50 \mathrm{Mpc}$ and the noise PSD of Advanced LIGO.

Assuming SNR $=5$ and considering only the post-merger signal, these results yield a detection horizon of about 13$27 \mathrm{Mpc}$, which reduces to $\simeq 9-19 \mathrm{Mpc}$ for randomly oriented sources. As summarized, for example, in Ref. [4], the detection rate is $\dot{N}=R N_{G}$, where $R$ is the coalescence rate of binaries per galaxy and $N_{G}$ is the number of galaxies accessible with a GW search. According to [4], $R \sim 100 \mathrm{MWEG}^{-1} \mathrm{Myr}^{-1}$ (MWEG = Milky Way Equivalent Galaxy) and $N_{G}$ can be estimated from Eq. (4) and Fig. 1 of [4] to be approximately 200 MWEG for horizons of $13-27 \mathrm{Mpc}^{6}$. In this way we derive a strict event rate of $\sim 0.01 \mathrm{yr}^{-1}$, which can, however, be increased to $\sim 0.1 \mathrm{yr}^{-1}$ if advanced detection techniques, such as the coherent waveburst algorithm, is employed [78].

We have also used the Fisher information matrix to estimate the uncertainties in the determination of the peak frequencies when a GW detection is made [26, 97]. Fisher matrix techniques are often used to forecast the precision of future experiments. Under certain standard assumptions, the Fisher matrix is the inverse of the covariance matrix, so it can be used to estimate the uncertainties on the parameters of the model. One of these assumptions is that, given a fiducial model, the Fisher matrix does not change too much if a different model is considered. Namely, the Fisher matrix error estimate is valid for models near the fiducial model [97].

In practice, we have followed [97] and estimated the errors in the determination of the peak frequency $f_{1}$ as (a similar expression can be obtained for the frequency $f_{2}$ by replacing

\footnotetext{
${ }^{6}$ In particular, we used the bottom curve of the two shown in Fig. 1 of [4], since it refers to the accessible blue-light luminosity for a given horizon distance, taking location and orientation averaging into account.
} 


\begin{tabular}{l|c|c|c|c|c}
\hline \hline Binary & GNH3-q10-M1300 & ALF2-q10-M1300 & H4-q10-M1300 & SLY-q10-M1300 & APR4-q10-M1300 \\
\hline GNH3-q10-M1300 & 0 & 31 & 7 & 78 & 74 \\
\hline ALF2-q10-M1300 & 18 & 0 & 25 & 32 & 32 \\
\hline H4-q10-M1300 & 10 & 42 & 0 & 64 & 85 \\
\hline SLY-q10-M1300 & 150 & 130 & 130 & 0 & 7 \\
\hline APR4-q10-M1300 & 140 & 90 & 190 & 11 & 0 \\
\hline \hline
\end{tabular}

TABLE IV. Errors $\delta f_{1}$ and $\delta f_{2}$ in the parameter estimation of $f_{1}$ and $f_{2}$ computed through the Fisher information matrix (see [58]). The SNRs used in this estimate are computed from the waveform starting $7.4 \mathrm{~ms}$ before the merger and ending $24.6 \mathrm{~ms}$ after the merger, assuming sources with optimal orientation at $50 \mathrm{Mpc}$ and the noise spectrum of Advanced LIGO. All values are expressed in Hz, with numbers above the diagonal referring to $\delta f_{1}$, and those below the diagonal referring to $\delta f_{2}$.

1 with 2 below)

$$
\begin{aligned}
& \delta f_{1} \equiv \sqrt{\overline{\left(\delta f_{1}\right)^{2}}}=\left|f_{1 \mathrm{~A}}-f_{1 \mathrm{~B}}\right| \times \\
& \left(4 \operatorname{Re} \int_{0}^{\infty} \frac{\left[\tilde{h}_{\mathrm{A}}\left(f^{\prime}\right)-\tilde{h}_{\mathrm{B}}\left(f^{\prime}\right)\right]\left[\tilde{h}_{\mathrm{A}}\left(f^{\prime}\right)-\tilde{h}_{\mathrm{B}}\left(f^{\prime}\right)\right]^{*}}{S_{h}\left(f^{\prime}\right)} d f^{\prime}\right)^{-1 / 2}
\end{aligned}
$$

where $f_{1 \mathrm{~A}}$ and $f_{1 \mathrm{~B}}$ are the $f_{1}$ frequencies of two models $\mathrm{A}$ and $\mathrm{B}, \tilde{h}_{\mathrm{A}}$ and $\tilde{h}_{\mathrm{B}}$ are the Fourier transforms of the waveforms of models A and B, respectively, and $S_{h}$ is the noise spectrum of Advanced LIGO. The denominator of expression (27) is the SNR of the difference of the signals of the two models.

Neglecting correlations between the peak frequencies and other parameters, we list in Table IV the errors estimated in this way for selected models, namely equal-mass models with the average gravitational mass at infinite separation $\bar{M} / M_{\odot}=$ 1.30 and all the EOSs we simulated. As mentioned, the Fisher matrix method is valid only for models near the correct model, so the values in Table IV for models whose frequencies are very different are not particularly meaningful and are reported only for completeness. In any case, the errors computed using only the post-merger phase are of the order of $1-10 \%$ and thus compatible with the estimates of [78].

\section{CONCLUSIONS}

Making use of a large number of accurate and fully generalrelativistic simulations of the inspiral and merger of BNSs with nuclear EOSs, we have illustrated in detail the spectral properties of the post-merger GW signal, extending and complementing the information already provided in [30] and in other papers that have looked at these properties [25, 26, 28, 31, 32]. More specifically, we have shown that correlations exist between the two largest peak frequencies in the PSD of the post-merger signal (i.e., $f_{1}, f_{2}$ ) and the physical properties of the NSs composing the binary system (i.e., mass, radius and their combinations). One of these correlations, namely, that of the low-frequency peak $f_{1}$ with compactness $\mathcal{C}$, is rather tight and has all the properties of being quasiuniversal; that is, it has a functional behavior that does not depend on the EOS. On the other hand, the high-frequency peak $f_{2}$ shows correlations, either with the average density or with the radius of a nonrotating star with a mass of $1.6 M_{\odot}$, that are far less tight and depend on the EOS.

We have shown that exploiting the information coming from both of these frequencies, together with the knowledge of the total mass of the binary, a potential detection of the post-merger GW signal at the large SNR ratio can set tight constraints on the EOS of matter at nuclear densities. While in principle a single observation would be sufficient, in practice the uncertainties in the measurements of the peaks and the errors in the fitting of the various correlations will require a number of positive and high-SNR detections for the robust identification of the EOS.

An interesting side product of this work is also the construction of a simple mechanical toy model that can explain rather intuitively the main spectral features of the post-merger signal and shed light on the physical interpretation of the origin of the various peaks. Despite its crudeness, the toy model can reproduce reasonably well the complex waveforms emitted right after the merger.

The exciting prospects opened in this work call for a number of future improvements. First, it remains to be determined how robust the results found here are when realistic spins in the NSs are accounted for, or when the dynamics is extended to an ideal-magnetohydrodynamics (ideal-MHD) description [96]. Second, the phenomenology discussed here will need to be studied also when fully three-dimensional EOSs are considered, or in more extreme regimes of unequalmass ratios. Third, the potential of the toy model presented here in predicting the post-merger signal needs to be further developed and validated. Last but not least, a proper assessment of the errors associated with the measurements of the stellar radii will require a complete Bayesian analysis of the probability that the constraints coming from the measurement of the two peak frequencies and of the total mass are satisfied simultaneously. Work in this direction is already in progress [94].

\section{ACKNOWLEDGMENTS}

We thank F. Galeazzi for help on the construction of the toy model and N. Stergioulas for useful discussions. Partial support comes from the DFG Grant SFB/Transregio 7 and by "NewCompStar", COST Action MP1304. K. T. is supported 
by the LOEWE-Program in HIC for FAIR. L. B. is supported in part by the JSPS Grant-in-Aid for Scientific Research C, No. 26400274. The simulations were performed on SuperMUC at LRZ-Munich, on Datura at AEI-Potsdam, and on LOEWE at CSC-Frankfurt.

\section{Appendix A: A MECHANICAL TOY MODEL FOR THE POST-MERGER SIGNAL}

We illustrate here the properties of a mechanical toy model that can be studied to reproduce many of the features of the spectral properties of the post-merger signal. Consider therefore a mechanical system composed of a disk of mass $M$ and radius $R$ rotating at a given angular frequency $\Omega(t)$, to which two spheres, each of mass $m / 2$, are connected (e.g., via a shaft), but are also free to oscillate via a spring that connects them (see Fig. 17). The spring has an elastic constant $k$ such that the oscillation frequency $\sqrt{k / m}$ is comparable with the rotation frequency $\Omega$.

In such a system, the two spheres will either approach each other, decreasing the moment of inertia of the system, or move away from each other, increasing the moment of inertia. Conservation of the total angular momentum will then lead to a variation of the rotation speed of the system and the generation of GWs with a precise spectrum.

Assuming that the spring will remain straight at all times and that the canonical coordinates and momenta are, respectively, $q^{i}$ and $\dot{q}^{i}$, the dynamics of the system with Lagrangian $L$ will be described by the Euler-Lagrange equations

$$
\frac{d}{d t}\left(\frac{\partial L}{\partial \dot{q}^{i}}\right)-\frac{\partial L}{\partial q^{i}}=\frac{\partial D}{\partial \dot{q}^{i}}
$$

where, $L=T-V, T$, and $V$ are the kinetic and potential energies, respectively, while $D$ accounts for dissipative effects. In a cylindrical coordinate system $(\varpi, \varphi, z)$ these quantities are expressed as

$$
\begin{aligned}
T & =\frac{1}{2} m\left[\dot{\varpi}^{2}+(\varpi \dot{\varphi})^{2}\right]+\frac{1}{2}\left(\frac{M R^{2}}{2}\right) \dot{\varphi}^{2}, \\
V & =2 k\left(\varpi-\varpi_{0}\right)^{2}, \\
D & =-b \dot{\varpi}^{2},
\end{aligned}
$$

where $\varpi_{0}$ is the natural displacement of the mass when $\Omega=0$, and $b$ is the damping coefficient. As a result, the Lagrangian of the system can be written as

$L=\frac{1}{2} m\left[\dot{\varpi}^{2}+(\varpi \dot{\varphi})^{2}\right]+\frac{1}{2}\left(\frac{M R^{2}}{2}\right) \dot{\varphi}^{2}-2 k\left(\varpi-\varpi_{0}\right)^{2}$.

We can then obtain the equation of motion for the radial displacement of the sphere $\varpi=\varpi(t)$ after computing that

$$
\begin{aligned}
\frac{\partial L}{\partial \dot{\varpi}} & =m \dot{\varpi}, & \frac{d}{d t}\left(\frac{\partial L}{\partial \dot{\varpi}}\right) & =m \ddot{\varpi}, \\
\frac{\partial L}{\partial \varpi} & =m \varpi \dot{\varphi}^{2}-4 k\left(\varpi-\varpi_{0}\right), & \frac{\partial D}{\partial \dot{\varpi}} & =-2 b \dot{\varpi} .
\end{aligned}
$$

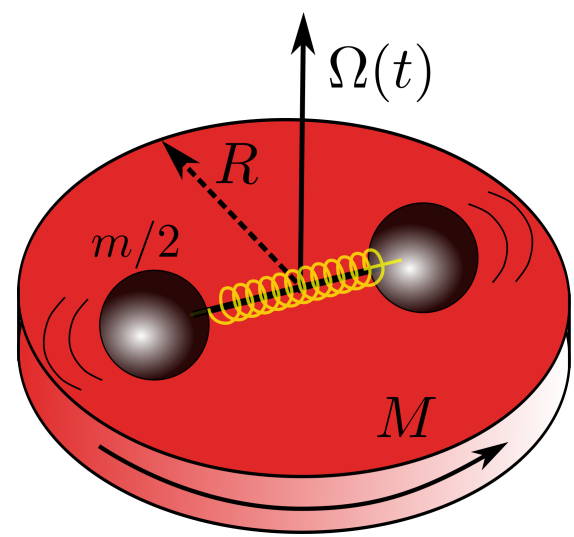

FIG. 17. Cartoon of the mechanical toy model composed of a disk of mass $M$ and radius $R$ rotating at frequency $\Omega(t)$. Two spheres, each of mass $m / 2$ are connected to the disk, but are also free to oscillate via a spring that connects them.

So as to obtain the following second-order differential equation:

$$
\ddot{\varpi}+\frac{4 k\left(\varpi-\varpi_{0}\right)}{m}-\varpi \dot{\varphi}^{2}+\frac{2 b \dot{\varpi}}{m}=0,
$$

Similarly, after using

$$
\begin{aligned}
\frac{\partial L}{\partial \dot{\varphi}} & =\left(\frac{M R^{2}}{2}+m \varpi^{2}\right) \dot{\varphi} \\
\frac{d}{d t}\left(\frac{\partial L}{\partial \dot{\varphi}}\right) & =\left(\frac{M R^{2}}{2}+m \varpi^{2}\right) \ddot{\varphi}+2 m \varpi \dot{\varpi} \dot{\varphi} \\
\frac{\partial L}{\partial \varphi} & =0=\frac{\partial D}{\partial \dot{\varphi}}
\end{aligned}
$$

we can express the equation of motion for the angular displacement of the sphere via the differential equation

$$
\ddot{\varphi}=-\frac{4 m \varpi \dot{\varpi} \dot{\varphi}}{M R^{2}+2 m \varpi^{2}} .
$$

Equation (A12) can be rewritten as

$$
\frac{d(\ln \dot{\varphi})}{d t}=-\frac{d}{d t}\left[\ln \left(\frac{M R^{2}}{2 m}+\varpi^{2}\right)\right],
$$

and can be integrated analytically to obtain

$$
\Omega(t) \equiv \dot{\varphi}=\frac{c_{1}}{\varpi^{2}+M R^{2} /(2 m)},
$$

where $c_{1}$ is an integration constant related to the total angular momentum $J_{\text {tot }}$, i.e., $c_{1}=J_{\text {tot }} / m$. By replacing $\dot{\varphi}$ in Eq. (A8) with the expression given in Eq. (A14), we can rewrite the differential equation (A8) as

$$
\ddot{\varpi}+\frac{4 k\left(\varpi-\varpi_{0}\right)}{m}-\left[\frac{c_{1}}{\varpi^{2}+M R^{2} /(2 m)}\right]^{2} \varpi+\frac{2 b \dot{\varpi}}{m}=0 .
$$


Equation (A15) can easily be integrated numerically to obtain $\varpi=\varpi(t)$ and hence $\Omega=\Omega(t)$ from Eq. (A14).

The top panel of Fig. 18 shows a typical solution for $\Omega(t)$ obtained with $M=10, m=10, k=0.25$, and $c_{1}=$ 25 , where the red solid line refers to an undamped system (i.e., with $b=0$ ), while the blue solid one to a damped system (i.e., with $b=0.25$ ).

As anticipated in Sec. V B, conservation of the total angular momentum implies that the evolution of the angular frequency of the system is bounded by two frequencies $\Omega_{1}$ and $\Omega_{3}$ (cf., dashed horizontal lines in the top panel of Fig. 18). These frequencies correspond respectively to the spinning frequency of the system when the spheres are at the largest separation (i.e., $\Omega_{1}$, when the moment of inertia is the largest) and to the spinning frequency when the spheres are at the smallest separation (i.e., $\Omega_{3}$, when the moment of inertia is the smallest). In the absence of dissipative forces, i.e., for $b=0$, the angular frequency simply oscillates between $\Omega_{1}$ and $\Omega_{3}$ (red solid line). Conversely, the oscillations are damped in the presence of a dissipative term, i.e., for $b \neq 0$, and the angular velocity asymptotes to a constant value, i.e., $\Omega(t) \rightarrow \Omega_{2}$ for $t \rightarrow \infty$, where $\Omega_{2} \sim \frac{1}{2}\left(\Omega_{1}+\Omega_{3}\right)$ for the parameters chosen here (blue solid line).

Because the time spent at a given frequency is $\tau_{\Omega}=$ $\Omega /(d \Omega / d t)$, more time is obviously spent at those frequencies where $d \Omega / d t \simeq 0$, i.e., $\Omega_{1}$ and $\Omega_{3}$. As a result, more power is expected to appear at these frequencies, hence producing a low-frequency peak around $\Omega_{1}$ and a high-frequency peak around $\Omega_{3}$. If dissipative processes are not present, the power is expected at the frequency $\Omega_{2}$ and at its overtones $\Omega_{n} \simeq(n / 2) \Omega_{2}$ for our reference choice of parameters, from which it follows that $\Omega_{2} \simeq \frac{1}{2}\left(\Omega_{1}+\Omega_{3}\right)$. This changes considerably if dissipative processes are present, as in this case most of the time is spent by the system around $\Omega_{2}$, so that most of the power in the PSD will appear around $\Omega_{2}$, with two main sidebands at $\Omega_{1}$ and $\Omega_{3}$.

We can validate this picture by computing the GW signal of the toy model in terms of the Newtonian quadrupole formula. To this end we need to calculate the relevant components of the quadrupole-moment tensor, which can be split into a timeindependent part relative to the rotating disk $\boldsymbol{I}^{\mathrm{d}}$ and a timedependent part relative to the oscillating spheres $\boldsymbol{I}^{\mathrm{s}}$, i.e.,

$$
I_{i j}(t)=I_{i j}^{\mathrm{d}}+I_{i j}^{\mathrm{s}}(t)
$$

The only nonzero components of $I_{i j}^{\mathrm{s}}(t)$ are

$$
\begin{aligned}
I_{x x}^{\mathrm{s}}(t) & =\left(\frac{m}{2}\right) \varpi^{2}(t)[1+\cos (2 \varphi(t))], \\
I_{y y}^{\mathrm{s}}(t) & =\left(\frac{m}{2}\right) \varpi^{2}(t)[1-\cos (2 \varphi(t))], \\
I_{x y}^{\mathrm{s}}(t) & =\left(\frac{m}{2}\right) \varpi^{2}(t) \sin (2 \varphi(t)) .
\end{aligned}
$$

Assuming an optimally oriented observer at distance $d$, the

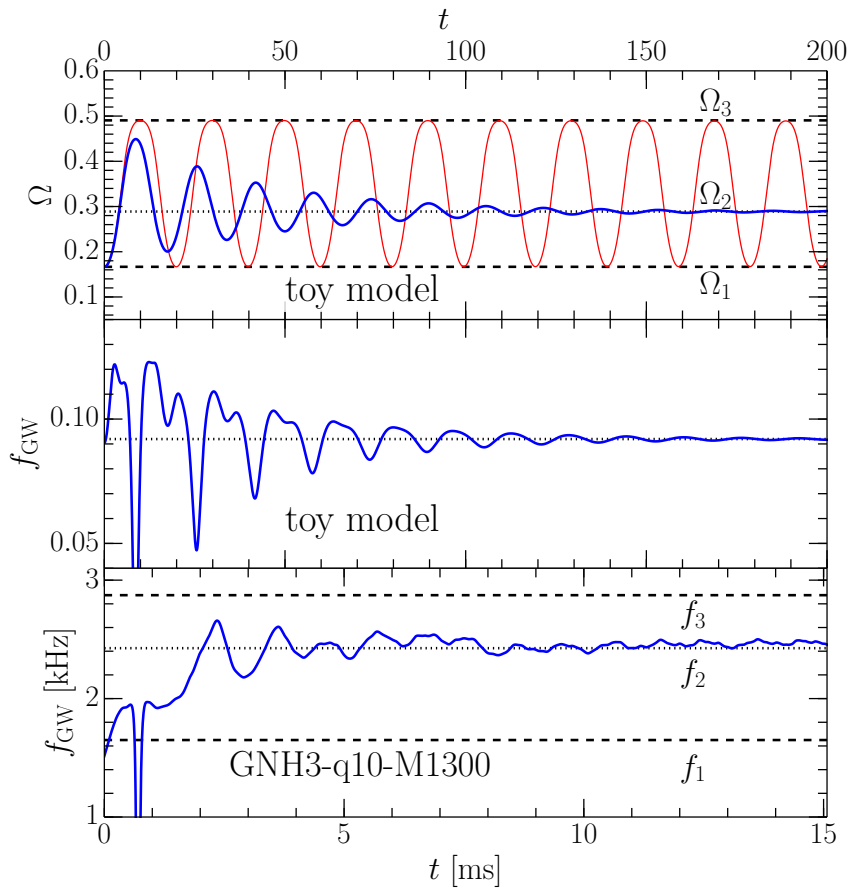

FIG. 18. Top panel: Time evolution in arbitrary units of the angular frequency $\Omega(t)$ in the mechanical toy model for a typical solution obtained with $M=10, m=10$, and $k=0.25$; the red solid line refers to an undamped system (i.e., with $b=0$ ), while the blue solid one to a damped system (i.e., with $b=0.25$ ). Note that in the first case $\Omega$ oscillates between $\Omega_{1}$ and $\Omega_{3}$ (black dashed lines), while in the second case it asymptotes to $\Omega_{2} \sim \frac{1}{2}\left(\Omega_{1}+\Omega_{3}\right)$ (black dotted line). Middle panel: Evolution of the instantaneous GW frequency as computed from the toy model. Bottom panel: Evolution of the instantaneous GW frequency $f_{\mathrm{GW}}$ for the binary GNH3-q10-M1300; cf., Fig. 10 for the actual GW strain.

GW strain in the two + and $\times$ polarizations is then given by

$$
\begin{aligned}
\left(\frac{d}{2}\right) h_{+}= & \frac{\ddot{I}_{x x}-\ddot{I}_{y y}}{2} \\
= & m\left[\left\{\dot{\varpi}^{2}+\varpi\left(\ddot{\varpi}-2 \varpi \Omega^{2}\right)\right\} \cos (2 \varphi)\right. \\
& \quad-\varpi(4 \dot{\varpi} \Omega+\varpi \dot{\Omega}) \sin (2 \varphi)], \\
\left(\frac{d}{2}\right) h_{\times}= & \ddot{I}_{x y} \\
= & m\left[\begin{array}{r}
\left.\dot{\varpi}^{2}+\varpi\left(\ddot{\varpi}-2 \varpi \Omega^{2}\right)\right\} \sin (2 \varphi) \\
\quad+\varpi(4 \dot{\varpi} \Omega+\varpi \dot{\Omega}) \cos (2 \varphi)],
\end{array}\right.
\end{aligned}
$$

where $I_{i j} \equiv I_{i j}-\frac{1}{3} \delta_{i j} \delta^{\ell m} I_{\ell m}$ is the reduced (trace-free) quadrupole moment tensor and $\delta_{i j}$ is the Kronecker delta.

The results of the GW signal computed with the toy model were already presented in Sec. VB, where it was remarked that despite the crudeness of the toy model, a good match appeared between the strain computed from the fully numerical simulations and the one coming from the toy model (cf., Fig. 10). Figure 18 offers another comparison between the GW computed from the toy model and those calculated 


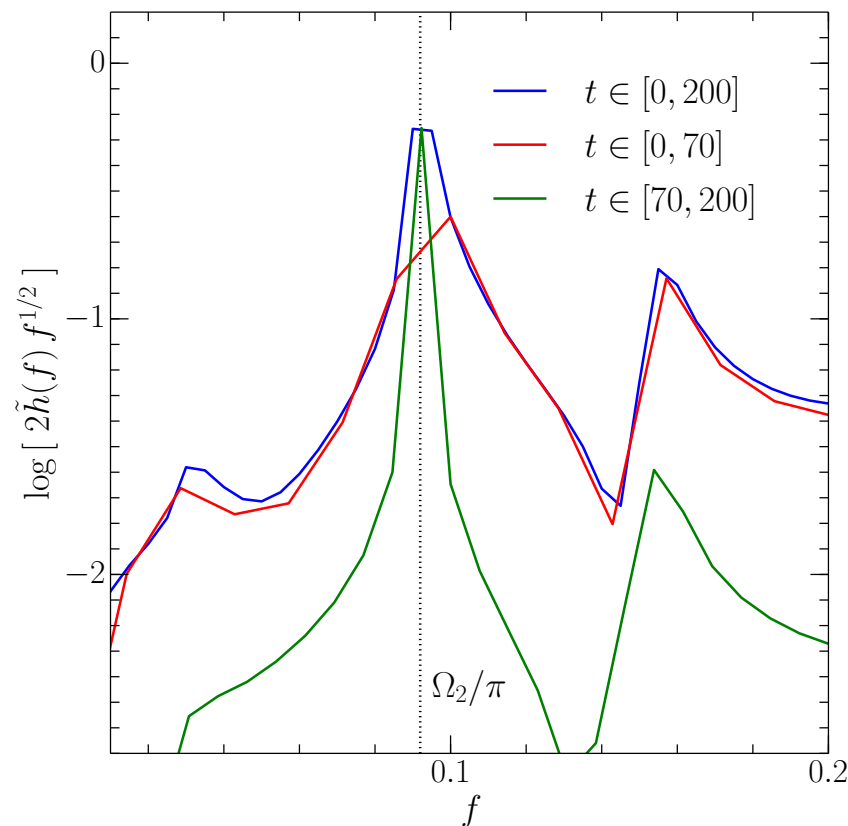

FIG. 19. PSDs $2 \tilde{h}(f) f^{1 / 2}$ of the GW signal produced by the toy model. The blue solid line refers to the full time series, while the other lines refer to signals where either the final part has been removed (red solid line) or the final one (green solid line). Shown with a vertical dashed line is the frequency $F_{2}=\Omega_{2} / \pi$. All units are arbitrary. from the numerical-relativity simulations. More specifically, the middle panel of Fig. 18 reports the instantaneous GW frequency $f_{\mathrm{GW}}$ of the + polarization (A14) computed for the toy model, while the bottom panel reports the corresponding instantaneous GW frequency as computed for the binary GNH3-q10-M1300. The similarities in the two instantaneous frequencies are a measure of the ability of the toy model to capture, especially for stiff EOSs, the essence of the GW emission right after the merger.

Finally, Fig. 19 reports the PSDs $2 \tilde{h}(f) f^{1 / 2}$ of the GW signal produced by the toy model via Eq. (A20) and for a damped oscillation with $b=0.25$. Adopting arbitrary units for the time, the blue solid line refers to the full time series, i.e., $t \in[0,200]$, the red one refers to a signal in which the final part has been removed, i.e., $t \in[0,70]$, while the green one refers to a signal in which the initial part has been removed, i.e., $t \in[70,200]$. As expected, in the case of the full time series, the PSD exhibits three main peaks at frequencies $F_{1}, F_{2}$, and $F_{3}$, with $F_{2}=\Omega_{2} / \pi \sim \frac{1}{2}\left(F_{1}+F_{3}\right)$. Furthermore, if the latter part of the signal is removed, then the power in the $F_{2}$ frequency is significantly reduced (red solid line). Similarly, for a signal that concentrates only on the decaying phase of the oscillations, the $F_{1}, F_{3}$ peaks are significantly suppressed (green solid line).
[1] G. M. Harry et al., Class. Quantum Grav. 27, 084006 (2010).

[2] T. Accadia et al., Class. Quantum Grav. 28, 114002 (2011).

[3] Y. Aso, Y. Michimura, K. Somiya, M. Ando, O. Miyakawa, T. Sekiguchi, D. Tatsumi, and H. Yamamoto, Phys. Rev. D 88, 043007 (2013).

[4] J. Abadie et al., Class. Quantum Grav. 27, 173001 (2010).

[5] R. Narayan, B. Paczynski, and T. Piran, Astrophysical Journal, Letters 395, L83 (1992).

[6] D. Eichler, M. Livio, T. Piran, and D. N. Schramm, Nature 340, 126 (1989).

[7] M. Shibata and K. Uryū, Phys. Rev. D 61, 064001 (2000).

[8] L. Baiotti, B. Giacomazzo, and L. Rezzolla, Phys. Rev. D 78, 084033 (2008).

[9] M. Anderson, E. W. Hirschmann, L. Lehner, S. L. Liebling, P. M. Motl, D. Neilsen, C. Palenzuela, and J. E. Tohline, Phys. Rev. D 77, 024006 (2008).

[10] Y. T. Liu, S. L. Shapiro, Z. B. Etienne, and K. Taniguchi, Phys. Rev. D 78, 024012 (2008).

[11] S. Bernuzzi, M. Thierfelder, and B. Brügmann, Phys. Rev. D 85, 104030 (2012).

[12] E. Berger, Annual Review of Astron. and Astrophys. 52, 43 (2014).

[13] C. Palenzuela, L. Lehner, M. Ponce, S. L. Liebling, M. Anderson, D. Neilsen, and P. Motl, Phys. Rev. Lett. 111, 061105 (2013).

[14] D. M. Siegel, R. Ciolfi, A. I. Harte, and L. Rezzolla, Phys. Rev. D R 87, 121302 (2013).

[15] K. Kiuchi, K. Kyutoku, Y. Sekiguchi, M. Shibata, and T. Wada,
Phys. Rev. D 90, 041502 (2014).

[16] L. Rezzolla, B. Giacomazzo, L. Baiotti, J. Granot, C. Kouveliotou, and M. A. Aloy, Astrophys. J. Letters 732, L6 (2011).

[17] V. Paschalidis, M. Ruiz, and S. L. Shapiro, arXiv:1410.7392 (2014).

[18] É. É. Flanagan and T. Hinderer, Phys. Rev. D 77, 021502 (2008).

[19] L. Baiotti, T. Damour, B. Giacomazzo, A. Nagar, and L. Rezzolla, Phys. Rev. Lett. 105, 261101 (2010).

[20] S. Bernuzzi, A. Nagar, M. Thierfelder, and B. Brügmann, Phys. Rev. D 86, 044030 (2012).

[21] D. Radice, L. Rezzolla, and F. Galeazzi, Mon. Not. R. Astron. Soc. L. 437, L46 (2014).

[22] D. Radice, L. Rezzolla, and F. Galeazzi, Class. Quantum Grav. 31, 075012 (2014).

[23] M. Shibata, Phys. Rev. Lett. 94, 201101 (2005).

[24] R. Oechslin and H. T. Janka, Phys. Rev. Lett. 99, 121102 (2007).

[25] A. Bauswein and H.-T. Janka, Phys. Rev. Lett. 108, 011101 (2012).

[26] A. Bauswein, H.-T. Janka, K. Hebeler, and A. Schwenk, Phys. Rev. D 86, 063001 (2012).

[27] K. Hotokezaka, K. Kiuchi, K. Kyutoku, T. Muranushi, Y.-i. Sekiguchi, M. Shibata, and K. Taniguchi, Phys. Rev. D 88, 044026 (2013).

[28] N. Stergioulas, A. Bauswein, K. Zagkouris, and H.-T. Janka, Mon. Not. R. Astron. Soc. 418, 427 (2011).

[29] A. Bauswein, N. Stergioulas, and H.-T. Janka, Phys. Rev. D 90 , 
023002 (2014).

[30] K. Takami, L. Rezzolla, and L. Baiotti, Phys. Rev. Lett. 113, 091104 (2014).

[31] J. S. Read, L. Baiotti, J. D. E. Creighton, J. L. Friedman, B. Giacomazzo, K. Kyutoku, C. Markakis, L. Rezzolla, M. Shibata, and K. Taniguchi, Phys. Rev. D 88, 044042 (2013).

[32] S. Bernuzzi, A. Nagar, S. Balmelli, T. Dietrich, and M. Ujevic, Phys. Rev. Lett. 112, 201101 (2014).

[33] URL http://astro.uni-frankfurt.de/ relastrowiki.

[34] L. Baiotti, B. Giacomazzo, and L. Rezzolla, Classical and Quantum Gravity 26, 114005 (2009).

[35] L. Baiotti, M. Shibata, and T. Yamamoto, Phys. Rev. D 82, 064015 (2010).

[36] D. Brown, P. Diener, O. Sarbach, E. Schnetter, and M. Tiglio, Phys. Rev. D 79, 044023 (2009).

[37] F. Löffler, J. Faber, E. Bentivegna, T. Bode, P. Diener, R. Haas, I. Hinder, B. C. Mundim, C. D. Ott, E. Schnetter, G. Allen, M. Campanelli, and P. Laguna, Class. Quantum Grav. 29, 115001 (2012).

[38] T. Nakamura, K. Oohara, and Y. Kojima, Progress of Theoretical Physics Supplement 90, 1 (1987).

[39] M. Shibata and T. Nakamura, Phys. Rev. D 52, 5428 (1995).

[40] T. W. Baumgarte and S. L. Shapiro, Phys. Rev. D 59, 024007 (1999).

[41] M. Alcubierre, B. Brügmann, P. Diener, M. Koppitz, D. Pollney, E. Seidel, and R. Takahashi, Phys. Rev. D 67, 084023 (2003).

[42] D. Pollney, C. Reisswig, L. Rezzolla, B. Szilágyi, M. Ansorg, B. Deris, P. Diener, E. N. Dorband, M. Koppitz, A. Nagar, and E. Schnetter, Phys. Rev. D 76, 124002 (2007).

[43] L. Baiotti, I. Hawke, P. J. Montero, F. Löffler, L. Rezzolla, N. Stergioulas, J. A. Font, and E. Seidel, Phys. Rev. D 71, 024035 (2005).

[44] L. Rezzolla, L. Baiotti, B. Giacomazzo, D. Link, and J. A. Font, Class. Quantum Grav. 27, 114105 (2010).

[45] P. Tsatsin and P. Marronetti, Phys. Rev. D 88, 064060 (2013).

[46] R. Donat and A. Marquina, Journal of Computational Physics 125, 42 (1996).

[47] A. Harten, P. D. Lax, and B. van Leer, SIAM Rev. 25, 35 (1983).

[48] P. Colella and P. R. Woodward, Journal of Computational Physics 54, 174 (1984).

[49] L. Rezzolla and O. Zanotti, Relativistic Hydrodynamics (Oxford University Press, Oxford, UK, 2013).

[50] W. Kastaun, F. Galeazzi, D. Alic, L. Rezzolla, and J. A. Font, Phys. Rev. D 88, 021501 (2013).

[51] E. Schnetter, S. H. Hawley, and I. Hawke, Class. Quantum Grav. 21, 1465 (2004).

[52] A. Akmal, V. R. Pandharipande, and D. G. Ravenhall, Phys. Rev. C 58, 1804 (1998).

[53] M. Alford, M. Braby, M. Paris, and S. Reddy, Astrophys. J. 629, 969 (2005).

[54] F. Douchin and P. Haensel, Astron. Astrophys. 380, 151 (2001).

[55] N. K. Glendenning and S. A. Moszkowski, Phys. Rev. Lett. 67, 2414 (1991).

[56] N. K. Glendenning, Astrophys. J. 293, 470 (1985).

[57] J. Antoniadis, P. C. C. Freire, N. Wex, T. M. Tauris, R. S. Lynch, M. H. van Kerkwijk, M. Kramer, C. Bassa, V. S. Dhillon, T. Driebe, J. W. T. Hessels, V. M. Kaspi, V. I. Kondratiev, N. Langer, T. R. Marsh, M. A. McLaughlin, T. T. Pennucci, S. M. Ransom, I. H. Stairs, J. van Leeuwen, J. P. W. Verbiest, and D. G. Whelan, Science 340, 1233232 (2013).

[58] J. S. Read, B. D. Lackey, B. J. Owen, and J. L. Friedman, Phys. Rev. D 79, 124032 (2009).
[59] URL http: / / www. lorene.obspm. fr.

[60] H.-T. Janka, T. Zwerger, and R. Mönchmeyer, Astron. Astrophys. 268, 360 (1993).

[61] T. Damour, A. Nagar, and L. Villain, Phys. Rev. D 85, 123007 (2012).

[62] D. Alic, C. Bona-Casas, C. Bona, L. Rezzolla, and C. Palenzuela, Phys. Rev. D 85, 064040 (2012).

[63] D. Alic, W. Kastaun, and L. Rezzolla, Phys. Rev. D 88, 064049 (2013).

[64] E. Gourgoulhon, P. Grandclément, K. Taniguchi, J.-A. Marck, and S. Bonazzola, Phys. Rev. D 63, 064029 (2001).

[65] J. N. Goldberg, A. J. MacFarlane, E. T. Newman, F. Rohrlich, and E. C. G. Sudarshan, J. Math. Phys. 8, 2155 (1967).

[66] K. Takami, L. Rezzolla, and L. Baiotti, Phys. Rev. Lett. 113, 091104 (2014).

[67] Advanced LIGO anticipated sensitivity curves, LIGO Document No. T0900288-v3.

[68] M. Punturo et al., Classical Quantum Gravity 27, 084007 (2010).

[69] M. Punturo et al., Class. Quantum Grav. 27, 194002 (2010).

[70] C. Messenger, K. Takami, S. Gossan, L. Rezzolla, and B. S. Sathyaprakash, Phys. Rev. X 4, 041004 (2014).

[71] F. J. Harris, Proceedings of the IEEE 66, 51 (1978).

[72] D. J. A. McKechan, C. Robinson, and B. S. Sathyaprakash, Classical and Quantum Gravity 27, 084020 (2010).

[73] S. Butterworth, Experimental Wireless and the Wireless Engineer 7, 536541 (1930).

[74] H. Shen, H. Toki, K. Oyamatsu, and K. Sumiyoshi, Nucl. Phys. A 637, 435 (1998).

[75] A. Bauswein, H. Janka, and R. Oechslin, Phys. Rev. D 82, 084043 (2010).

[76] L. D. Landau and E. M. Lifshitz, Mechanics, Volume 1 (Pergamon Press, Oxford, 1976).

[77] O. Zanotti, J. A. Font, L. Rezzolla, and P. J. Montero, Mon. Not. R. Astron. Soc. 356, 1371 (2005).

[78] J. Clark, A. Bauswein, L. Cadonati, H.-T. Janka, C. Pankow, and N. Stergioulas, Phys. Rev. D 90, 062004 (2014).

[79] K. Yagi and N. Yunes, Science 341, 365 (2013).

[80] K. Yagi and N. Yunes, Phys. Rev. D 88, 023009 (2013).

[81] M. Urbanec, J. C. Miller, and Z. Stuchlík, Mon. Not. R. Astron. Soc. 433, 1903 (2013).

[82] D. D. Doneva, S. S. Yazadjiev, N. Stergioulas, and K. D. Kokkotas, Astrophys. J. Letters 781, L6 (2014).

[83] B. Haskell, R. Ciolfi, F. Pannarale, and L. Rezzolla, Mon. Not. R. Astron. Soc. Letters 438, L71 (2014).

[84] G. Pappas and T. A. Apostolatos, Phys. Rev. Lett. 112, 121101 (2014).

[85] S. Chakrabarti, T. Delsate, N. Gürlebeck, and J. Steinhoff, Phys. Rev. Lett. 112, 201102 (2014).

[86] L. C. Stein, K. Yagi, and N. Yunes, Astrophys. J. 788, 15 (2014).

[87] A. Maselli, V. Cardoso, V. Ferrari, L. Gualtieri, and P. Pani, Phys. Rev. D 88, 023007 (2013).

[88] K. Chatziioannou, K. Yagi, and N. Yunes, Phys. Rev. D 90, 064030 (2014).

[89] Y.-H. Sham, L.-M. Lin, and P. T. Leung, Astrophys. J. 781, 66 (2014).

[90] P. Pani and E. Berti, Phys. Rev. D 90, 024025 (2014).

[91] D. D. Doneva, S. S. Yazadjiev, K. V. Staykov, and K. D. Kokkotas, Phys. Rev. D 90, 104021 (2014).

[92] K. Yagi, K. Kyutoku, G. Pappas, N. Yunes, and T. A. Apostolatos, Phys. Rev. D 89, 124013 (2014).

[93] Y.-H. Sham, T. K. Chan, L.-M. Lin, and P. T. Leung, The Astrophysical Journal 798, 121 (2015). 
[94] C. Messenger, L. Rezzolla, and K. Takami, in preparation (2015).

[95] S. Bernuzzi, T. Dietrich, W. Tichy, and B. Brügmann, Phys. Rev. D 89, 104021 (2014).
[96] B. Giacomazzo, L. Rezzolla, and L. Baiotti, Phys. Rev. D 83, 044014 (2011).

[97] J. S. Read, C. Markakis, M. Shibata, K. Uryū, J. D. E. Creighton, and J. L. Friedman, Phys. Rev. D 79, 124033 (2009). 